\title{
The role of microstructure and processing on the proton conducting properties of gadolinium-doped barium cerate
}

\author{
Sossina M. Haile ${ }^{\text {a) }}$ \\ Department of Material Science, California Institute of Technology, Pasadena, California 91125 \\ David L. West ${ }^{\mathrm{b})}$ and John Campbell \\ Department of Materials Science and Engineering, University of Washington, \\ Seattle, Washington 98195-2120 \\ (Received 19 August 1997; accepted 12 January 1998) \\ The influence of grain boundary conductivity and microstructure on the electrical \\ properties of $\mathrm{BaCe}_{0.85} \mathrm{Gd}_{0.15} \mathrm{O}_{3-\delta}$ have been examined. Grain sizes were varied by \\ sintering at various temperatures. Impedance data were analyzed using the brick layer \\ model, and some new consequences of this model are presented. The specific grain \\ boundary conductivity exhibits an activation energy of $\sim 0.7 \mathrm{eV}$, and for similar \\ processing routes, is independent of grain size. An isotope effect was observed, indicating \\ that protons (or deuterons) are the mobile species. TEM investigations showed the \\ intergranular regions to be free of any glassy phase that could account for the differences \\ in bulk and grain boundary properties. Single-crystal fibers, grown by a modified \\ float zone process, were notably barium deficient, and exhibited a low conductivity, \\ comparable to that of polycrystalline $\mathrm{Ba}_{0.96} \mathrm{Ce}_{0.85} \mathrm{Gd}_{0.15} \mathrm{O}_{3-\delta}$.
}

\section{INTRODUCTION}

Proton conduction in doped perovskite oxides has been the subject of extensive investigation ever since Iwahara and co-workers demonstrated the utility of such materials in hydrogen sensors and other solid state ionic devices. ${ }^{1}$ Proton incorporation into such materials has been generally recognized to occur by a two-step process. ${ }^{1-3}$ The first, the introduction of trivalent ions on the normally quatravalent ion site (e.g., replacement of $\mathrm{Ce}^{4+}$ in $\mathrm{BaCeO}_{3}$ with $\mathrm{Gd}^{3+}$ ), creates vacant oxygen sites, or, in oxidizing atmospheres, creates electronic holes. The second, the exposure of the material to $\mathrm{H}_{2} \mathrm{O}$ or $\mathrm{H}_{2}$ containing atmospheres, leads to the occupation of previously vacant oxygen sites by hydroxyl groups and the uptake of the remaining protons at other oxygen sites, or (again, for highly oxidized atmospheres) to the exchange of electronic holes with protons.

In contrast to the general consensus on the mechanism of bulk proton incorporation, there are unresolved questions with respect to the chemical and electrical properties of the grain boundary in these materials. Indeed, it has been proposed that the grain boundaries in barium cerate, in particular, are responsible for the overall high conductivity of this material. Such a conclusion would naturally drive the development of nanocrystalline materials. In the present work we demon-

\footnotetext{
a) Author to whom correspondence should be addressed.

${ }^{b}$ Present address: Department of Materials Science and Engineering, University of Illinois, Urbana-Champagne, Illinois 61801.
}

strate, on the basis of microstructural investigations by transmission and scanning electron microscopy, and electrical characterization by impedance spectroscopy of both polycrystalline and single-crystal materials, that the grain boundaries in barium cerate do not provide a high conductivity pathway for proton transport. In combination with our earlier work describing the variation in conductivity with slight variations in stoichiometry, ${ }^{4}$ we conclude that differences in composition produce much greater changes in the overall conductivity of Gd-doped barium cerate than do variations in microstructure.

\section{BACKGROUND}

\section{A. Proton incorporation}

The introduction of trivalent dopants into (Cedeficient) $\mathrm{BaCeO}_{3}$ is normally assumed to occur as per Eq. (1) (written in Kroeger-Vink notation), a mechanism that introduces oxygen vacancies.

$$
2 \mathrm{Ce}_{\mathrm{Ce}}^{x}+\mathrm{O}_{\mathrm{O}}^{x}+\mathrm{Gd}_{2} \mathrm{O}_{3} \longrightarrow 2 G d_{\mathrm{Ce}}^{\prime}+V_{\mathrm{O}}^{\bullet}+2 \mathrm{CeO}_{2} \text {. }
$$

Subsequent exposure to humid atmospheres is presumed to lead to the incorporation of protons as per Eq. (2).

$$
\mathrm{H}_{2} \mathrm{O} \text { (gas) }+V_{\mathrm{O}}^{\bullet \bullet}+\mathrm{O}_{\mathrm{O}} \longrightarrow 2 \mathrm{OH}_{\mathrm{O}}^{\bullet} \text {. }
$$

Thus, two protons (or one water molecule) can, in principle, be incorporated for each dopant ion added to the system.

The protons introduced according to Eq. (2) are not bound to any particular oxygen ion, but are rather free 


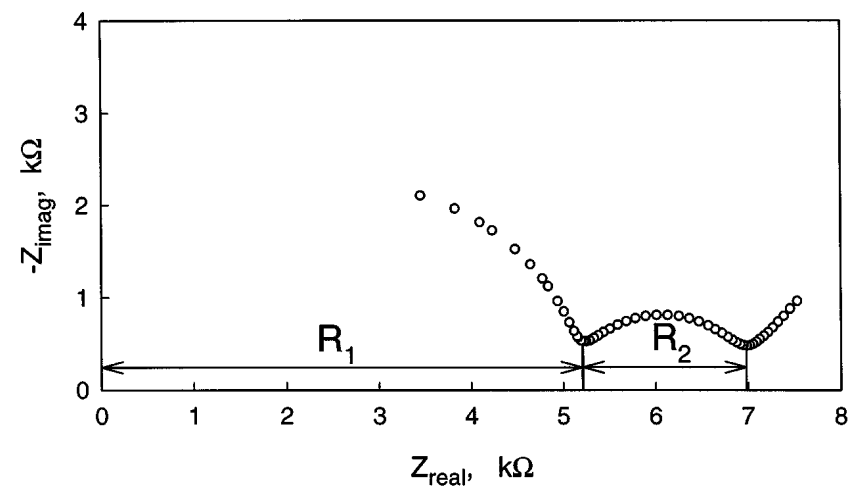

FIG. 1. A typical Nyquist plot for a polycrystalline material. The real and imaginary components of impedance are plotted as parametric functions of frequency. Data collected from $\mathrm{BaCe}_{0.85} \mathrm{Gd}_{0.15} \mathrm{O}_{3-\delta}$, over the frequency range $100 \mathrm{~Hz}$ to $1 \mathrm{MHz}$ with an applied voltage of $0.9 \mathrm{~V}$ and in an $\mathrm{H}_{2} \mathrm{O}$-saturated argon atmosphere. (Frequency increases from right to left.)

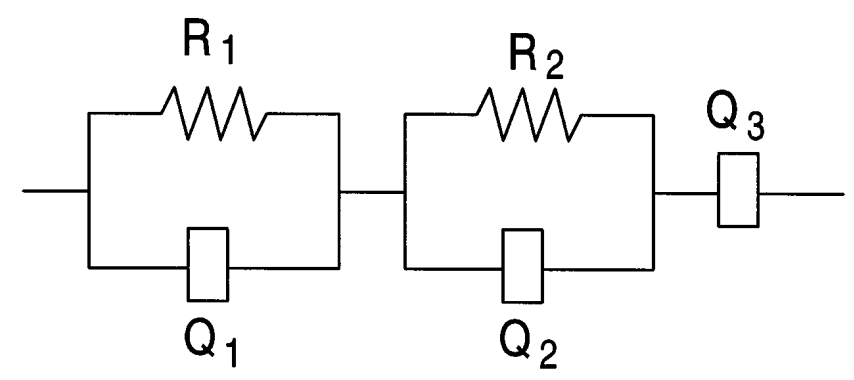

FIG. 2. A circuit of resistors, $R$, and constant phase elements, $Q$, that has the impedance shown in Fig. 1.

to migrate from one ion to the next. This easy migration results in the high proton conductivity observed in doped perovskite oxides.

\section{B. Grain boundary phases}

The possibility that an amorphous, Ba-rich phase exists at the grain boundaries of doped barium cerate was first proposed by Kreuer et al. ${ }^{5}$ Such a phase was expected to appear if some fraction of the trivalent dopant ions, e.g., $\mathrm{Gd}$, were to be incorporated into the structure of the perovskite, e.g., $\mathrm{BaCeO}_{3}$, on the divalent (barium) site rather than the quatravalent (cerium) site. As a result of dopant incorporation onto the "wrong" site, the material would essentially contain an excess of barium as demonstrated in Eq. (3).

$$
2 \mathrm{Ba}_{\mathrm{Ba}}+\mathrm{Gd}_{2} \mathrm{O}_{3} \longrightarrow \underset{\mathrm{Ba}}{2 \mathrm{Gd}^{\circ}}+\mathrm{O}_{\mathrm{i}}{ }^{\prime \prime}+2 \mathrm{BaO}_{\text {excess }},
$$

or

$$
\begin{aligned}
2 \mathrm{Ba}_{\mathrm{Ba}}+\mathrm{V}_{\mathrm{O}}^{\bullet \bullet}+\mathrm{Gd}_{2} \mathrm{O}_{3} \longrightarrow & 2 \mathrm{Gd}_{\mathrm{Ba}}^{\bullet} \\
+ & \mathrm{O}_{\mathrm{o}}{ }^{x}+2 \mathrm{BaO}_{\text {excess }} .
\end{aligned}
$$

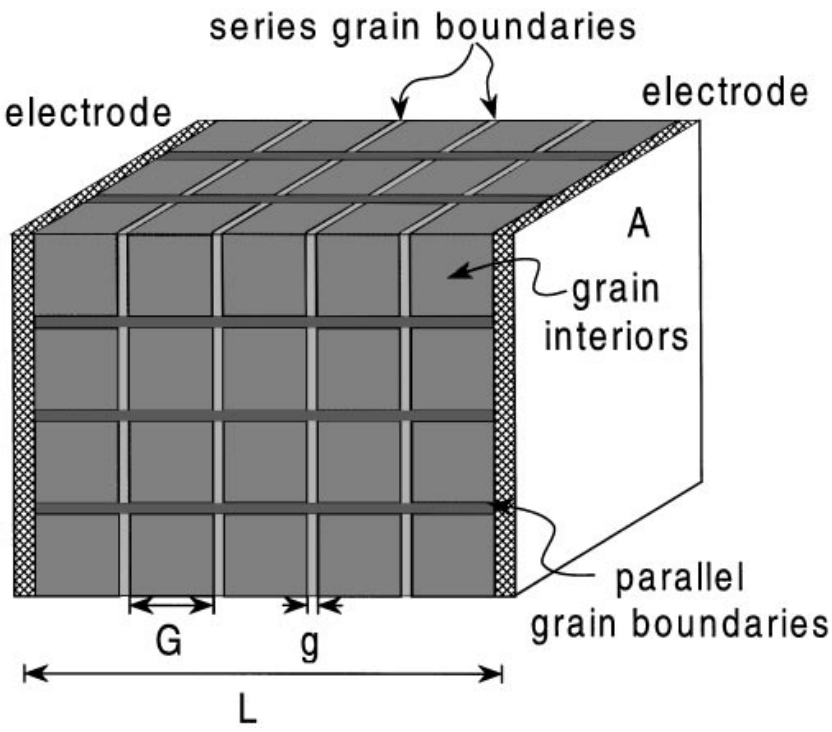

FIG. 3. "Brick Layer" model of a polycrystalline material. Grains are assumed to be cube-shaped, and grain boundaries to exist as flat layers between grains.

It is this excess barium oxide that was believed to reside at grain boundaries, and furthermore provide a pathway for fast proton transport. It is noteworthy that dopant ion incorporation by Eq. (3) also results in a concentration of oxygen vacancies that is lower than if Gd incorporation took place only on the intended Ce site and has, therefore, been used to explain differences in water uptake results as reported by different authors. ${ }^{6}$ Thus, knowledge of the grain boundary state is crucial to understanding both the bulk and grain boundary properties. As part of the present work, we have investigated polycrystalline Gd-doped barium cerate by transmission electron microscopy to elucidate the nature of the grain boundary regions.

\section{The brick layer model: New implications}

Impedance spectroscopy is a highly useful technique for studying the conductivity of ionic conductors in polycrystalline form, not only because the need for nonblocking electrodes is eliminated, but also because the resistances due to grain interiors and that due to grain boundaries can, in many cases, be measured independently. From these data and knowledge of the sample dimensions, the bulk (or grain interior) conductivity can be extracted in a rather straightforward manner. In order to determine grain boundary conductivity, in contrast, one requires some microstructural/electrical model as well as additional microstructural information, specifically, the average grain size and the average grain boundary thickness. While the procedures for calculating grain interior and grain boundary conductivities form the "brick layer model" discussed here are known (see, for example Refs. 7-10), they are reviewed here to enable 


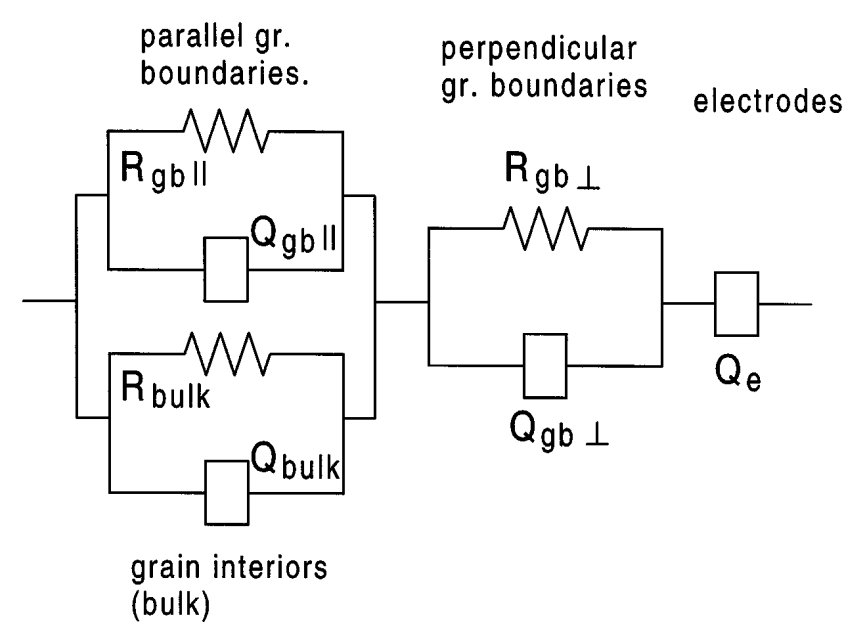

(a)

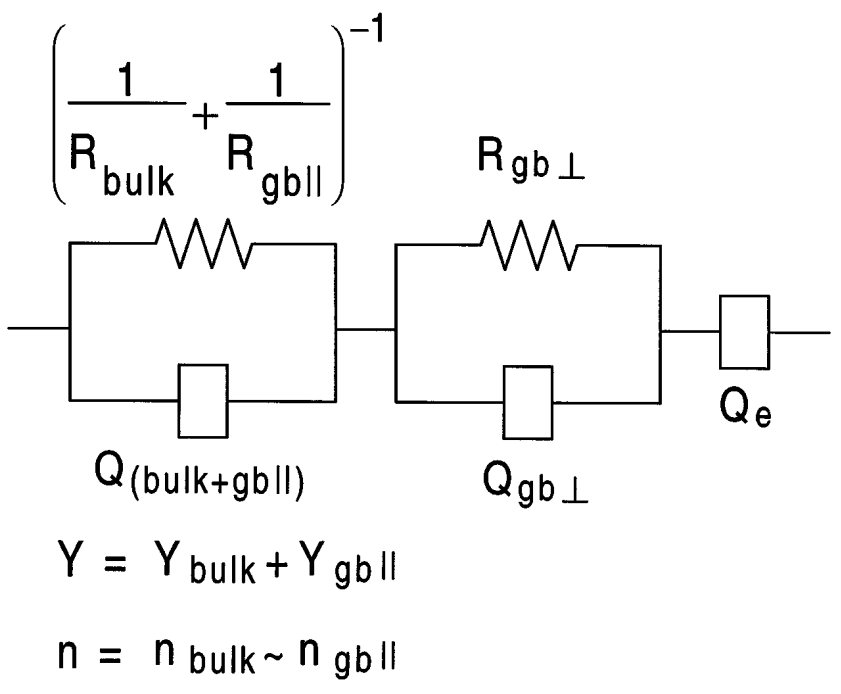

(b)

FIG. 4. Equivalent circuit models of a polycrystalline material with the brick layer microstructure. (a) Each component explicitly included in the circuit; (b) simplified equivalent circuit in which components in parallel have been lumped together. Note the similarity to Fig. 2.

a discussion of the impact of microstructure on the form of the Nyquist $\left(-Z_{I}\right.$ vs $\left.Z_{R}\right)$ plot, and of the full extent of the information that can be extracted directly from the electrical data.

A Nyquist representation of the impedance data of many polycrystalline materials exhibits an arc at high frequency, a second arc at lower frequencies, and a linear portion at the lowest frequencies (Fig. 1). In the absence of any knowledge of the properties or microstructural characteristics of the material, the impedance data can be fit to an equivalent circuit model containing one $Q$ element and two $(R Q)$ subcircuits ( $R$ and $Q$ in parallel) that are in series with one another, Fig. 2, where $R=$ resistor (with impedance $Z_{R}=R$ ) and $Q=$ constant phase element (with

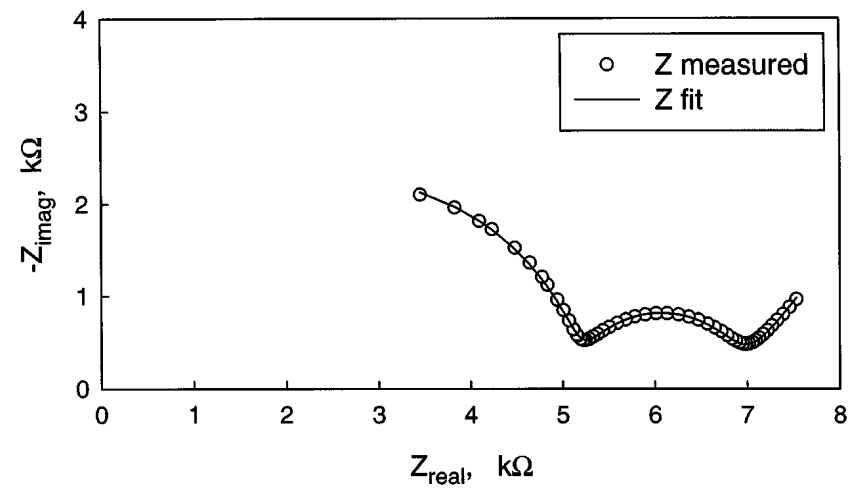

(a)

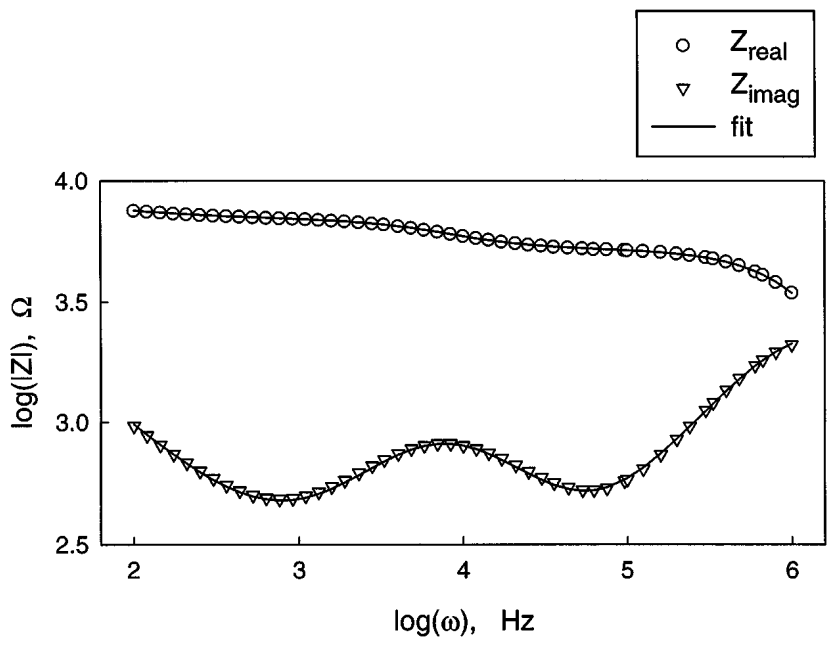

(b)

FIG. 5. A comparison of the measured and fit impedance for the data shown in Fig. 1. Fit parameters given in text. Data plotted in the (a) Nyquist representation; and (b) Bode representation.

impedance $Z_{Q}=\left[Y(j \omega)^{n}\right]^{-1},{ }^{11}$ where $j=\sqrt{-1}, \omega=$ frequency, $Y$ and $n$ are constants, and $n$ ranges between 0 and 1). Experimentally, the exponent $n$ characterizing the constant phase elements of the $(R Q)$ subcircuits are found to be rather close to 1 , and thus these elements behave much like capacitors, and we refer to $Y$ as the pseudo-capacitance. The frequency at the apex of each of the arcs in Fig. 1 corresponds to the characteristic frequency, $\omega_{0}$, of each of the $(R Q)$ subcircuits and is given by $(1 / Y R)^{1 / n} \cdot{ }^{11}$ If these frequencies are sufficiently different from one another and $n$ is close to 1 , the resistance of each of the subcircuits is given by the distance across the real axis that each arc extends, as shown in Fig. 1. In addition, the equivalent capacitances, $C$, of each subcircuit is given by $\left(R \omega_{0}\right)^{-1}$.

The two quantities of greatest interest are the resistances, $R_{1}$ and $R_{2}$, associated with each of the $(R Q)$ subcircuits. In order to correlate these with physically meaningful quantities, we model a polycrystalline material to consist of cube-shaped grains with grain bound- 




FIG. 6. X-ray diffraction patterns of ceramic powder with nominal composition $\mathrm{BaCe}_{0.85} \mathrm{Gd}_{0.15} \mathrm{O}_{3-\delta}$ postcalcination; (a) batch-1 material, containing a small amount of $\mathrm{BaGd}_{2} \mathrm{O}_{4}$; (b) batch-2 material, containing a small amount of $\mathrm{BaCO}_{3}$. Peaks due to impurity phases indicated with an asterisk.

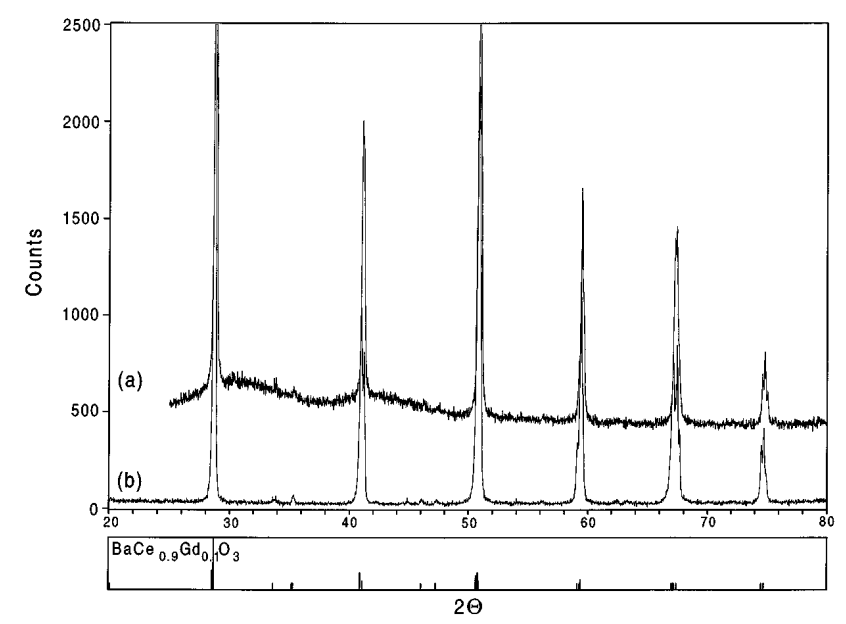

FIG. 7. X-ray powder diffraction patterns of sintered, Gd-doped barium cerate showing the absence of secondary phases: (a) $\mathrm{BaCe}_{0.9^{-}}$ $\mathrm{Gd}_{0.1} \mathrm{O}_{3-\delta}$; (b) $\mathrm{BaCe}_{0.85} \mathrm{Gd}_{0.15} \mathrm{O}_{3-\delta}$.

aries both in parallel with and perpendicular to the direction of the applied field (Fig. 3). This "brick layer" model, was first proposed by Beekmans and Heyne, ${ }^{12}$ and further discussed by van Dijk and Burggraaf. ${ }^{13}$ While these earlier works considered some limiting cases individually, we follow the generalized approach proposed by Nafe. ${ }^{8}$ The relevant geometric parameters are defined as $L=$ sample length, $A=$ sample crosssectional area, $G=$ edge length (or diameter) of the grains, and $g=$ grain boundary thickness.

For $g \ll G$, the total length and area of all perpendicular (or series) grain boundaries is given by:

$$
\begin{aligned}
& L_{\perp}=(g / G) L, \\
& A_{\perp}=A .
\end{aligned}
$$

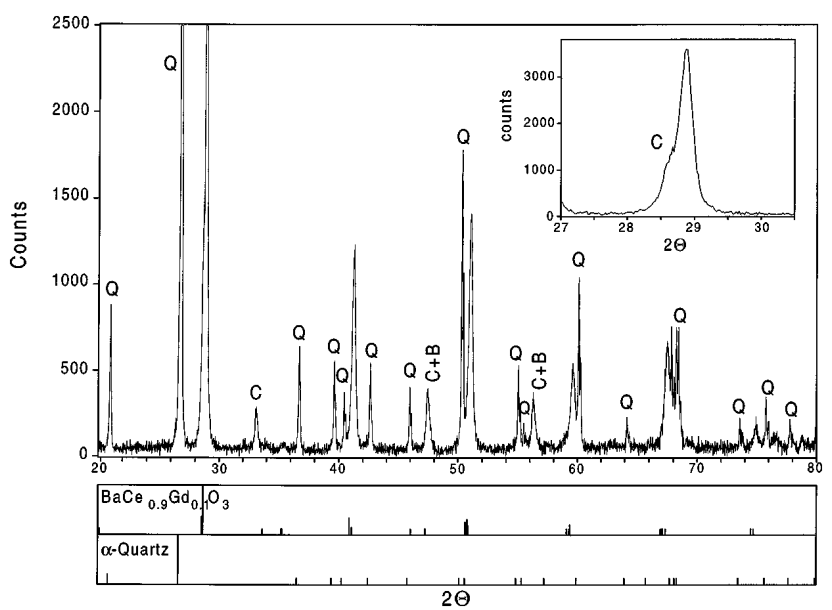

FIG. 8. X-ray powder diffraction pattern of single crystal fiber with nominal composition $\mathrm{BaCe}_{0.85} \mathrm{Gd}_{0.15} \mathrm{O}_{3-\delta}$. Peaks due to $\alpha$-quartz, used as an internal $2 \theta$ standard, and to $\mathrm{CeO}_{2}$, present as an impurity phase, marked with the letters $Q$ and $C$, respectively. (Background subtracted.)

Similarly, the total length and area of all parallel grain boundaries are given by:

$$
\begin{aligned}
& L_{\|}=L, \\
& A_{\|}=(2 g / G) A .
\end{aligned}
$$

If, in contrast, the grain boundary thickness were comparable in magnitude to the grain boundary edge length, the more general relationships presented by $\mathrm{Nafe}^{8}$ would more accurately give the total lengths and areas occupied by grain interiors and by parallel and perpendicular grains boundaries.

Assuming that each component of the material behaves as an $(R Q)$ subcircuit, as suggested by the experimentally obtained impedance data, the equivalent circuit model of an ideal polycrystalline material is as shown in Fig. 4(a). There are three subcircuits in series with one another. The first contains the $(R Q)$ components of grain interiors and of parallel grain boundaries, the second contains the $(R Q)$ components of only series grain boundaries, and the third a constant phase element describing the electrode response.

The equivalent circuit of Fig. 4(a) can be simplified, as is done in Fig. 4(b), by combining the two resistances that are in parallel into a single resistor, the inverse of which is given by

$$
1 / R_{g b \|}+1 / R_{\text {bulk }} .
$$

Similarly, the two constant phase elements can be combined into a single $Q$, which, assuming the $n$ 's are similar, has a total pseudo-capacitance of

$$
Y_{g b \|}+Y_{\text {bulk }} .
$$

To this point, we have not established which of the physical processes of the brick layer model correspond 
TABLE I. Composition of batch-2 samples of $\mathrm{BaCe}_{0.85} \mathrm{Gd}_{0.15} \mathrm{O}_{3-\delta}$ sintered at several different temperatures, in both grain interior and grain boundary regions. Number in parentheses indicates uncertainty in last digit(s).

\begin{tabular}{|c|c|c|c|c|c|c|}
\hline \multirow[b]{2}{*}{$T_{s}$} & \multicolumn{3}{|c|}{ Interior } & \multicolumn{3}{|c|}{ Boundary } \\
\hline & at. $\% \mathrm{Ba}$ & at. $\% \mathrm{Ce}$ & at. $\% \mathrm{Gd}$ & at. $\% \mathrm{Ba}$ & at. $\% \mathrm{Ce}$ & at. $\% \mathrm{Gd}$ \\
\hline 1700 & $19.2(9)$ & $17.0(8)$ & $4.2(2)$ & $19.4(9)$ & $16.8(8)$ & $4.4(2)$ \\
\hline 1600 & $19.3(9)$ & $17.0(8)$ & $4.2(2)$ & $19.4(9)$ & $16.8(8)$ & $4.3(2)$ \\
\hline 1500 & $19.5(9)$ & $16.8(8)$ & $4.3(2)$ & $19.4(9)$ & $16.8(8)$ & $4.3(2)$ \\
\hline 1400 & 19.2(9) & $16.9(8)$ & $4.3(2)$ & 19.3(9) & $16.9(8)$ & $4.3(2)$ \\
\hline average & $19.3(9)$ & $16.9(8)$ & $4.3(2)$ & $19.4(9)$ & $16.8(8)$ & $4.3(2)$ \\
\hline expected & 20.3 & 17.3 & 3.05 & 20.3 & 17.3 & 3.05 \\
\hline
\end{tabular}

to the high and low frequency responses observed in the experimentally obtained impedance plot. We proceed by making the usual assumption that the low to mid-frequency arc is associated with the response of grain boundaries in series with grain interiors, and that the high-frequency arc corresponds to the combined response of grain interiors and parallel grain boundaries. The basis for this assumption is discussed below.

Taking the grains and grain boundaries to have the specific conductivities of $\sigma_{\text {bulk }}$ and $\sigma_{g b}$, respectively, and operating under the assumption that transport in parallel and series grain boundaries occurs by the same mechanism, yields

$$
\frac{1}{R_{1}}=\frac{1}{R_{g b \|}}+\frac{1}{R_{\text {bulk }}}=\frac{A}{L}\left\{\frac{2 g}{G} \sigma_{g b}+\sigma_{\text {bulk }}\right\},
$$

and

$$
\frac{1}{R_{2}}=\frac{1}{R_{g b \perp}}=\frac{A}{L}\left\{\frac{G}{g} \sigma_{g b}\right\} .
$$

Defining $\sigma_{i}=L / A\left\{1 / R_{i}\right\}$ allows us to rewrite Eqs. (4) and (5) in terms of conductivity:

$$
\sigma_{1}=\left\{\frac{2 g}{G} \sigma_{g b}+\sigma_{\text {bulk }}\right\}
$$

and

$$
\sigma_{2}=\left\{\frac{G}{g} \sigma_{g b}\right\} .
$$

At first glance it appears that, while $R_{1}$ and $R_{2}$ can be obtained from a Nyquist plot, neither $\sigma_{g b}$ or $\sigma_{\text {bulk }}$ can be determined from an impedance measurement and a knowledge of macroscopic sample dimensions. The problem of how to interpret the impedance spectrum becomes more tractable if we assess, under various limiting conditions, the impact of the relative magnitudes of $\sigma_{g b}$ and $\sigma_{\text {bulk }}$ on the relative magnitudes of $R_{1}$ and $R_{2}$, and thereby on the Nyquist plots. The ratio $R_{2}$ to $R_{1}$ can be obtained from the ratio of $\sigma_{1}$ to $\sigma_{2}$ :

$$
\frac{\sigma_{1}}{\sigma_{2}}=\frac{R_{2}}{R_{1}}=2\left(\frac{g}{G}\right)^{2}+\left(\frac{g}{G}\right) \frac{\sigma_{\text {bulk }}}{\sigma_{g b}}
$$

and the reflects the fact that the relative resistance of $R_{1}$ to $R_{2}$ is dependent on both physical $\left(\sigma_{\text {bulk }} / \sigma_{g b}\right)$ and microstructural $(g / G)$ properties.

\section{Case (1): $\sigma_{\text {bulk }}>\sigma_{g b}$}

For the situation where $\sigma_{\text {bulk }}>\sigma_{g b}$ and a material has a typical microstructure with $g \ll G$, Eq. (8) reduces to

$$
\frac{\sigma_{1}}{\sigma_{2}}=\frac{R_{2}}{R_{1}}=\left(\frac{g}{G}\right) \frac{\sigma_{\text {bulk }}}{\sigma_{g b}},
$$

and, significantly, Eq. (6) reduces to

$$
\sigma_{1}=\sigma_{\text {bulk }} .
$$

Equation (10) is the usual assumption made in interpreting impedance spectra and measuring bulk conductivity. Current flow, in materials for which $\sigma_{\text {bulk }}>\sigma_{g b}$ is true, will follow grain interiors where it can, and pass through relatively high resistance grain boundaries only as a means of moving from one grain to the next. Significant deviation from the simplification of Eq. (1) occurs only when $\sigma_{g b} \gg \sigma_{\text {bulk }}$. Even if grain boundary and bulk conductivity were comparable, $\sigma_{g b} \sim \sigma_{\text {bulk }}$, the measured value of $\sigma_{1}$ would be only about $2 \%$ greater than expected from Eq. (10), for a microstructure in which $g / G=10^{-2}$. Physically, this reflects the fact that, even if grain boundaries were to present a relatively easy pathway for ion transport, the area presented by grain boundaries in parallel to grains is small, and ions consequently would utilize the larger area pathway offered by the grain interiors.

The Nyquist plots of materials with $\sigma_{\text {bulk }}>\sigma_{g b}$ will exhibit two distinguishable arcs if (i) $R_{1}$ and $R_{2}$ are comparable in magnitude and (ii) the characteristic frequencies of the two regions of the material are significantly different. The requirement that $R_{1}$ and $R_{2}$ be comparable, that is, be at least within two to three orders of magnitude of one another, reflects experimental realities: a small arc in a Nyquist plot may not be discernible if it resides next to a large arc that has 1000 times the radius. From Eq. (9) it is evident that materials with typical microstructures, $g / G<1$ (and 


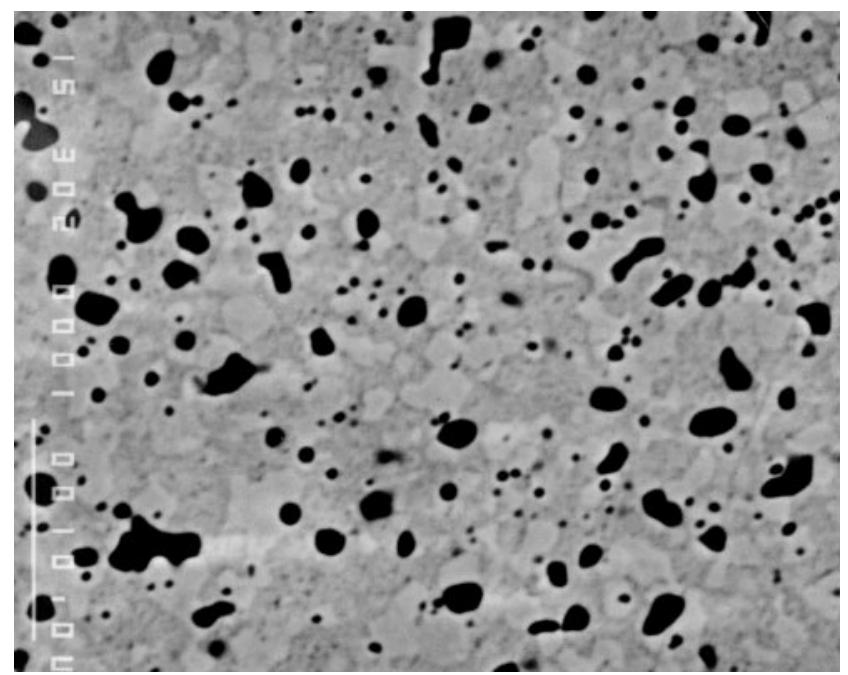

(a)

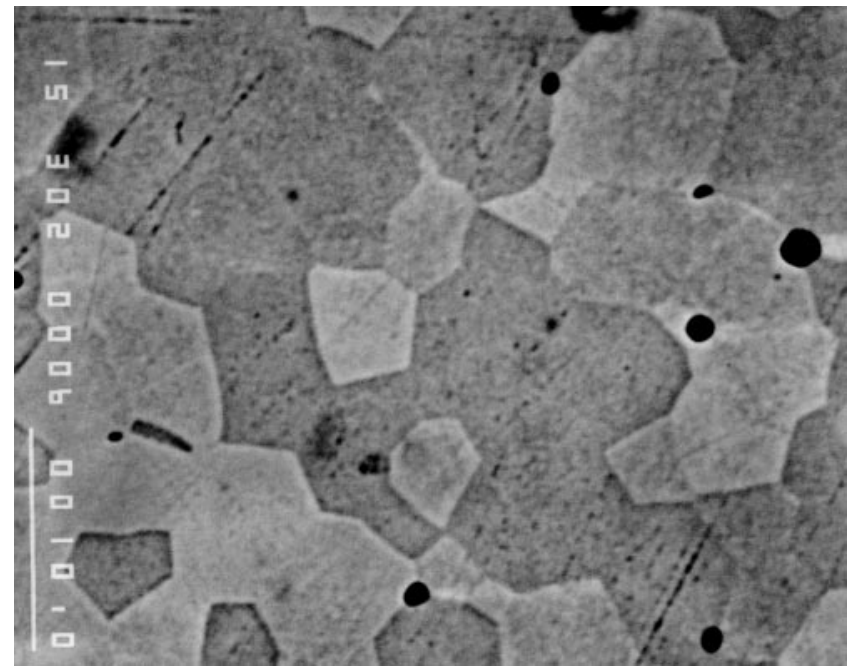

(c)

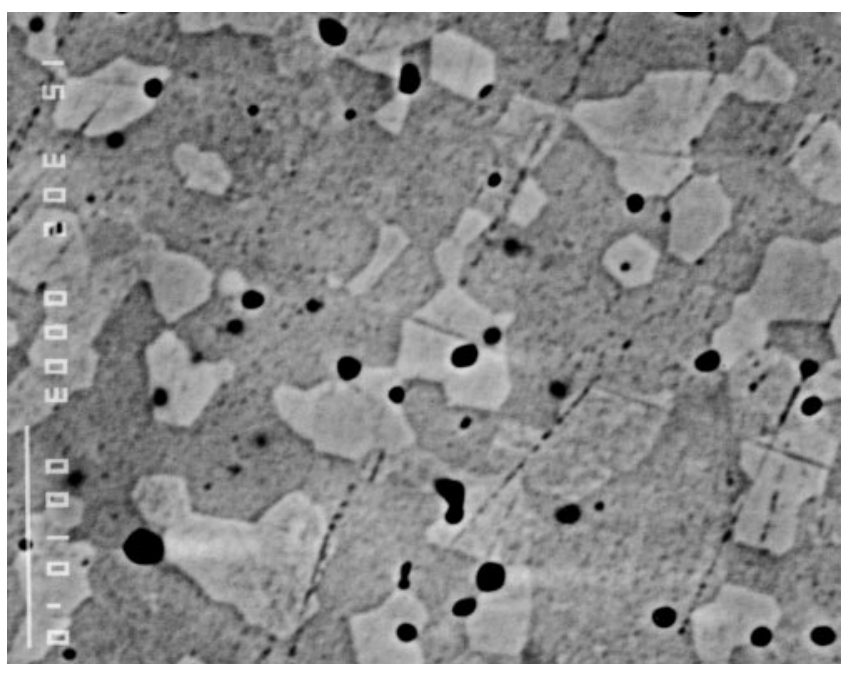

(b)

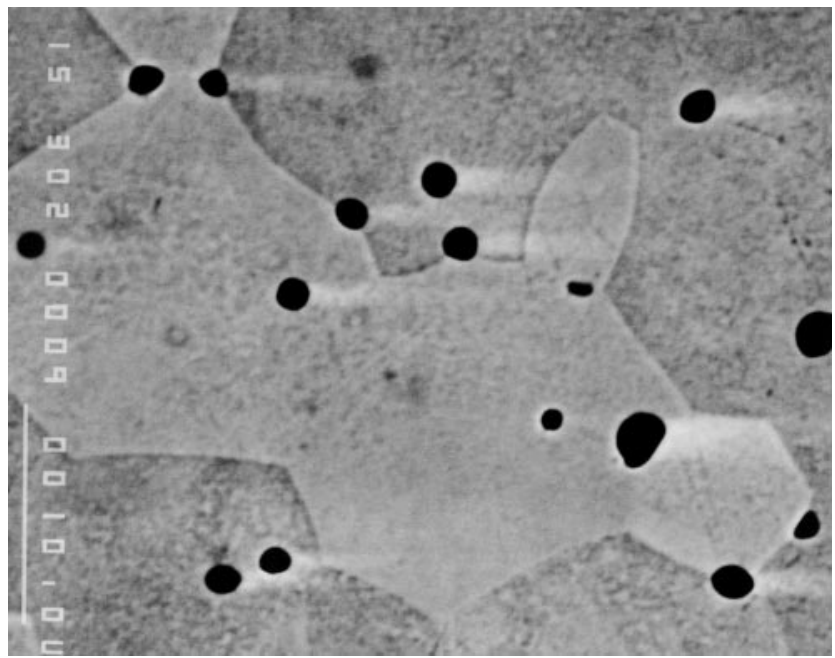

(d)

FIG. 9. Scanning electron micrographs of batch-2 samples of $\mathrm{BaCe}_{0.85} \mathrm{Gd}_{0.15} \mathrm{O}_{3-\delta}$ sintered at different temperatures. Bar on left-hand side of each figure corresponds to a length of $10 \mu \mathrm{m}$ : (a) $1400{ }^{\circ} \mathrm{C}$, (b) $1500{ }^{\circ} \mathrm{C}$, (c) $1600{ }^{\circ} \mathrm{C}$, and (d) $1700{ }^{\circ} \mathrm{C}$.

$\sigma_{\text {bulk }} / \sigma_{g b}>1$ ), will have $0.01<R_{1} / R_{2}<100$ for a wide range of conditions, and thus the first condition will be met for a wide range of combinations of $g / G$ and $\sigma_{\text {bulk }} / \sigma_{g b}$.

The characteristic frequency of a material or a region of a material, $\omega_{0}$ is, as implied above, given by $1 / R C$. Because $R$ and $C$ depend on geometric parameters in an inverse manner, $\omega_{0}$ is an intrinsic material property, independent of geometric considerations, and given by $1 / \rho \epsilon \epsilon_{0}$, where $\rho=$ resistivity $=1 / \sigma, \epsilon=$ relative dielectric constant, and $\epsilon_{0}=$ permittivity of free space. The question of whether $\omega_{0, g b}$ and $\omega_{0, \text { bulk }}$ are significantly different from one another reduces to the question of whether or not the products $\left(\rho_{g b} \epsilon_{g b}\right)$ and $\left(\rho_{\text {bulk }} \epsilon_{\text {bulk }}\right)$ differ significantly. While resistivities of materials cover many orders of magnitude, dielectric constants do not vary significantly. Thus, it is reasonable to make the usual assumption that $\epsilon_{\text {bulk }} \sim \epsilon_{g b},{ }^{7}$ and the question reduces even further to whether or not $\rho_{g b}$ and $\rho_{\text {bulk }}$ are significantly different from one another. For a material in which $\sigma_{\text {bulk }}>\sigma_{g b}$, by definition $\rho_{g b}>\rho_{\text {bulk }}$, which in turn implies $\omega_{0, g b}<\omega_{0, \text { bulk }}$. Consequently, two arcs will indeed be present in the Nyquist plot, and that at lower frequency will indeed correspond to the response of grain boundaries. Strictly speaking the characteristic frequency of the high-frequency arc is determined by the combined dielectric response of grain interiors and parallel grain boundaries (as well as their resistances). 


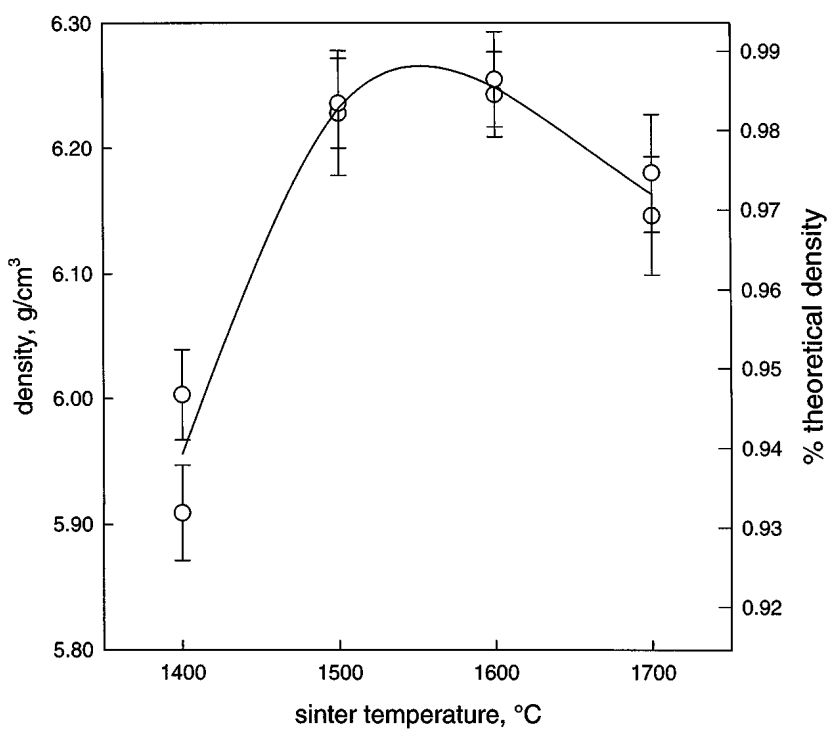

FIG. 10. The density of batch-2 samples of $\mathrm{BaCe}_{0.85} \mathrm{Gd}_{0.15} \mathrm{O}_{3-\delta}$ as a function of sintering temperature. Error bars represent uncertainty in the measurement of macroscopic dimension. Solid line is a guide to the eye.

However, because parallel grain boundaries have an unfavorable geometry (small area, long length), their contribution to the capacitance (assuming $\epsilon_{g b} \sim \epsilon_{\text {bulk }}$ ) is negligible. Surprisingly, it is common to justify the identification of the low frequency arc in a double-arc Nyquist plot as a (series) grain boundary response on the basis of geometric considerations (e.g., Ref. 14). While $C_{1}>C_{2}$ is generally true as a result of the large total area and short length of grain boundaries, the characteristic frequency, as stated above, is independent of geometry because $R C=\rho \epsilon \epsilon_{0}$.

While the measurement of $\sigma_{\text {bulk }}$ in materials of typical microstructures is straightforward if $\sigma_{\text {bulk }}>\sigma_{g b}$ [Eq. (10)], measurement of $\sigma_{g b}$ requires knowledge of the grain size and grain boundary thickness [Eq. (7)]. In the absence of microstructural observations that directly provide the values of $g$ and $G$, the ratio, $g / G$, can be estimated from the dielectric response of the material. Each of the $(R Q)$ subcircuits has a capacitance, $C$, that, as described above, can be obtained from a knowledge of the resistance and the characteristic frequency, or, of the resistance and the two parameters, $Y$ and $n$, that characterize the constant phase element. Specifically,

$$
C=\frac{1}{R \omega_{0}}=Y^{(1 / n)} R^{(1 / n-1)} .
$$

As mentioned above, because of the unfavorable geometry of parallel grain boundaries, the dielectric constant $C_{1}$, associated with the high frequency arc, should be almost entirely due to grain interiors, whereas $C_{2}$, the dielectric constant associated with the lower frequency arc, should be due entirely to perpendicular (or series)

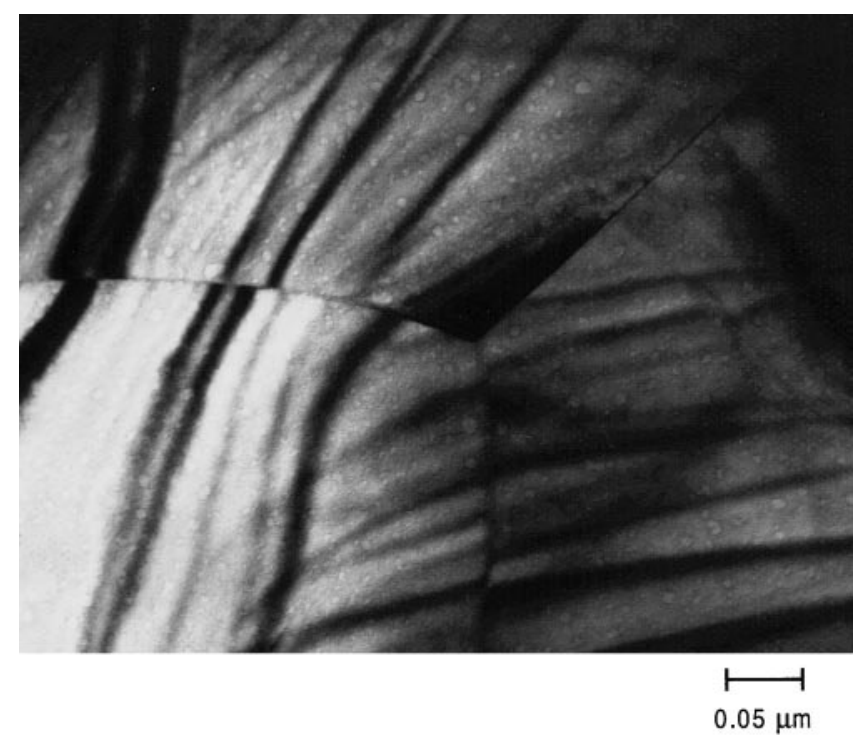

(a)

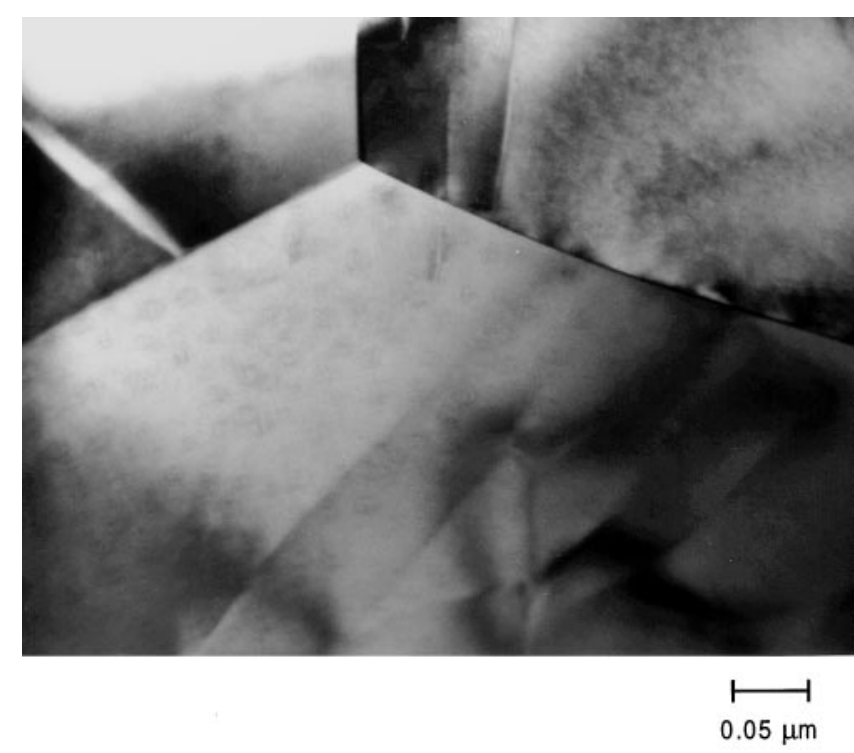

(b)

FIG. 11. Transmission electron micrographs of batch-1 samples of Gd-doped barium cerate, showing grain boundaries to be free of any amorphous phase (a) $\mathrm{BaCe}_{0.9} \mathrm{Gd}_{0.1} \mathrm{O}_{3-\delta}$ and (b) $\mathrm{BaCe}_{0.85} \mathrm{Gd}_{0.15} \mathrm{O}_{3-\delta}$.

grain boundaries. Thus,

$$
C_{1} \approx C_{\mathrm{bulk}}=\frac{A}{L} \epsilon_{\mathrm{bulk}} \epsilon_{0}
$$

and

$$
C_{2} \approx C_{g b \perp}=\frac{A}{L} \frac{G}{g} \epsilon_{g b} \epsilon_{0} .
$$

Because the dielectric properties of the bulk and grain boundaries are often similar, i.e., $\epsilon_{g b} \sim \epsilon_{\text {bulk }}$, then

$$
\frac{C_{1}}{C_{2}}=\frac{g}{G} \text {. }
$$



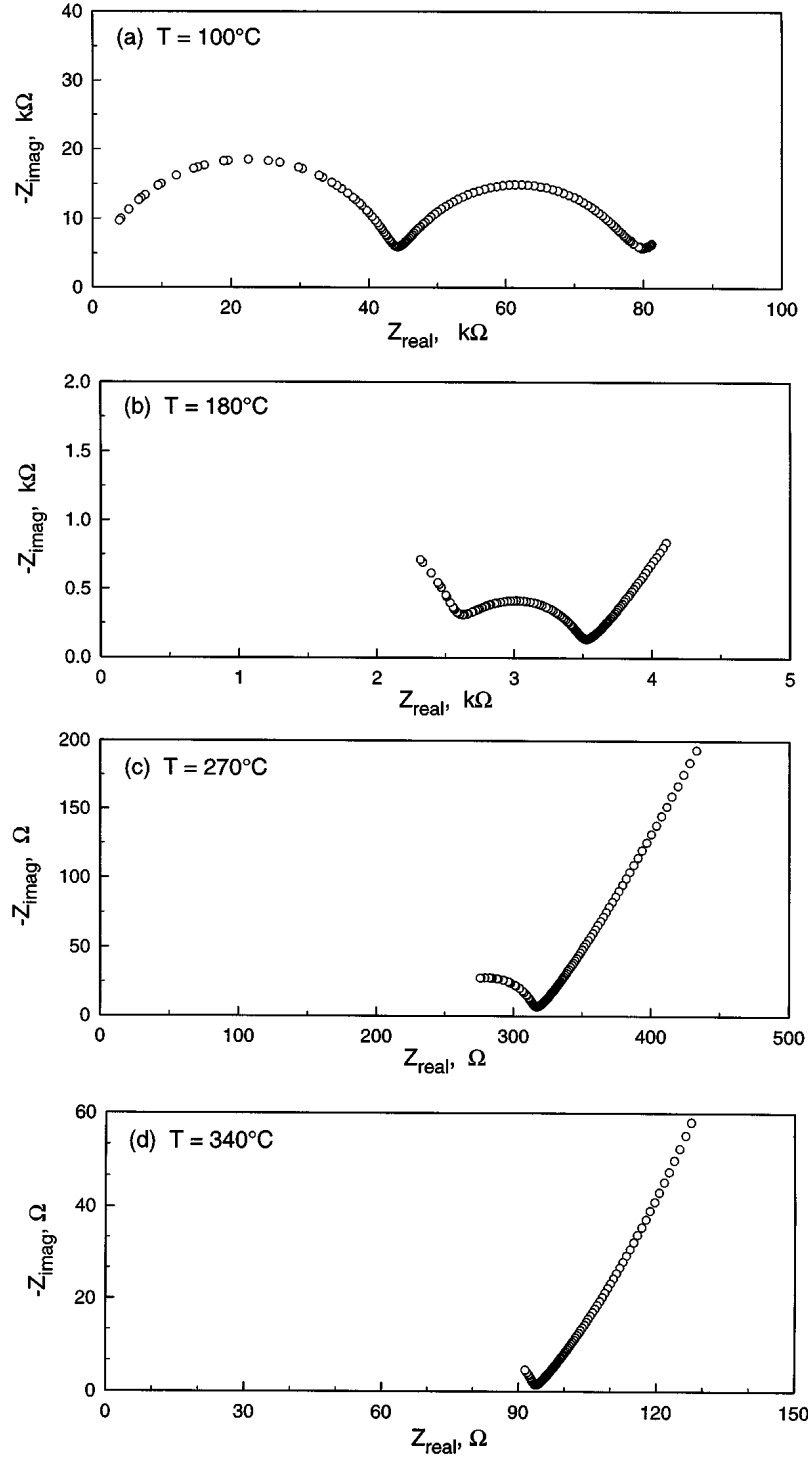

FIG. 12. Nyquist impedance plots obtained for batch-2 samples of $\mathrm{BaCe}_{0.85} \mathrm{Gd}_{0.15} \mathrm{O}_{3-\delta}$ sintered at $1400{ }^{\circ} \mathrm{C}$. Temperature of the measurement as indicated. Atmosphere is $\mathrm{H}_{2} \mathrm{O}$-saturated argon. Note that both $R_{1}$ and $R_{2}$ can be measured in (a), (b), and (c), whereas only the total resistance, $R_{1}+R_{2}$, can be measured in (d).

It is, therefore, entirely possible to measure both specific grain interior (or bulk) conductivity and specific grain boundary conductivity from a single impedance measurement, even in the absence of microstructural observations, so long as the frequency range utilized accurately yields all relevant parameters.

\section{Case (2): $\sigma_{g b}>\sigma_{\text {bulk }}$}

For those materials in which $\sigma_{g b}>\sigma_{\text {bulk }}$ and which have typical microstructures, $g / G$ no greater than $10^{-2}$, no simplification to Eq. (8) can be made. In order to determine the form of the Nyquist plot, we examine instead Eq. (4) for three subcases: (a) $\sigma_{g b} / \sigma_{\text {bulk }} \gg 1 / 2$
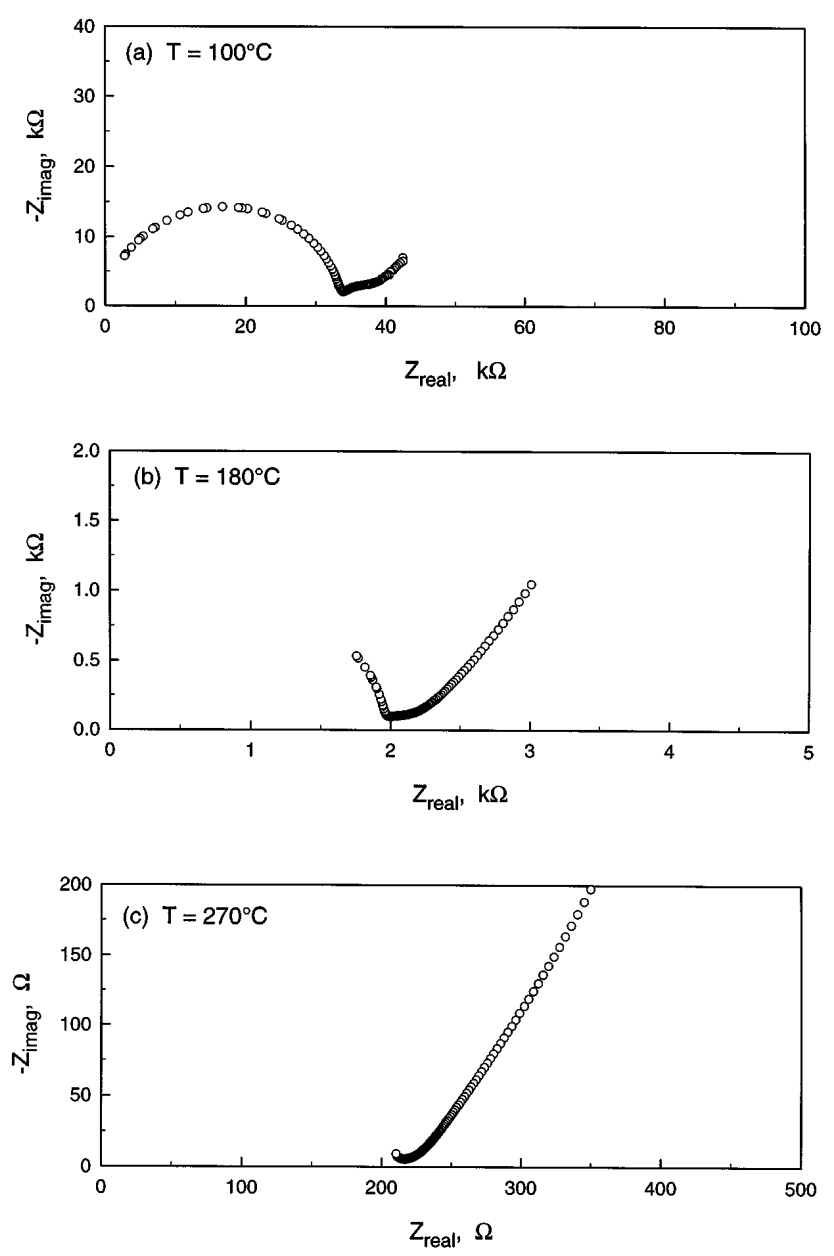

FIG. 13. (a-c) Nyquist impedance plots obtained for batch-2 samples of $\mathrm{BaCe}_{0.85} \mathrm{Gd}_{0.15} \mathrm{O}_{3-\delta}$ sintered at $1700{ }^{\circ} \mathrm{C}$. Temperature of the measurement as indicated. Atmosphere is $\mathrm{H}_{2} \mathrm{O}$-saturated argon. Unlike the situation reflected in Fig. 12, $R_{2}$ becomes negligible in (c) such that the total resistance is approximately $R_{1}$.

$G / g$. The term $R_{g b \|}$ will dominate $R_{1}$, and transport along parallel grain boundaries will be preferred to that through grain interiors; (b) $\sigma_{g b /} \sigma_{\text {bulk }} \sim 1 / 2 \mathrm{G} / g$. The terms $R_{g b \|}$ and $R_{\text {bulk }}$ are comparable in magnitude, and transport both along parallel grain boundaries and through grain interiors takes place; (c) $1 / 2(G / g) \sigma_{\text {bulk }}>$ $\sigma_{g b}>\sigma_{\text {bulk }}$. The resistance $R_{1}$ will, for the most part, be dominated by $R_{\text {bulk }}$ [the approximations of Eqs. (9) and (10) are valid], despite the greater specific resistance of grain interiors. This results from the more favorable geometry offered by grain interiors relative to the narrow and long parallel grain boundaries. Assuming, as was done above, that the dielectric constants of the bulk material and of its grain boundaries are approximately equal and that $n$ is close to 1 , the capacitance of the first $R Q$ circuit [Fig. 4(b)], for any of the subcases, is again given by $C_{1} \sim C_{\text {bulk }}$. The resistance, $R_{1}$, is within a factor of 2 of $R_{g b \|}$ for subcases (a) and (b), whereas it 


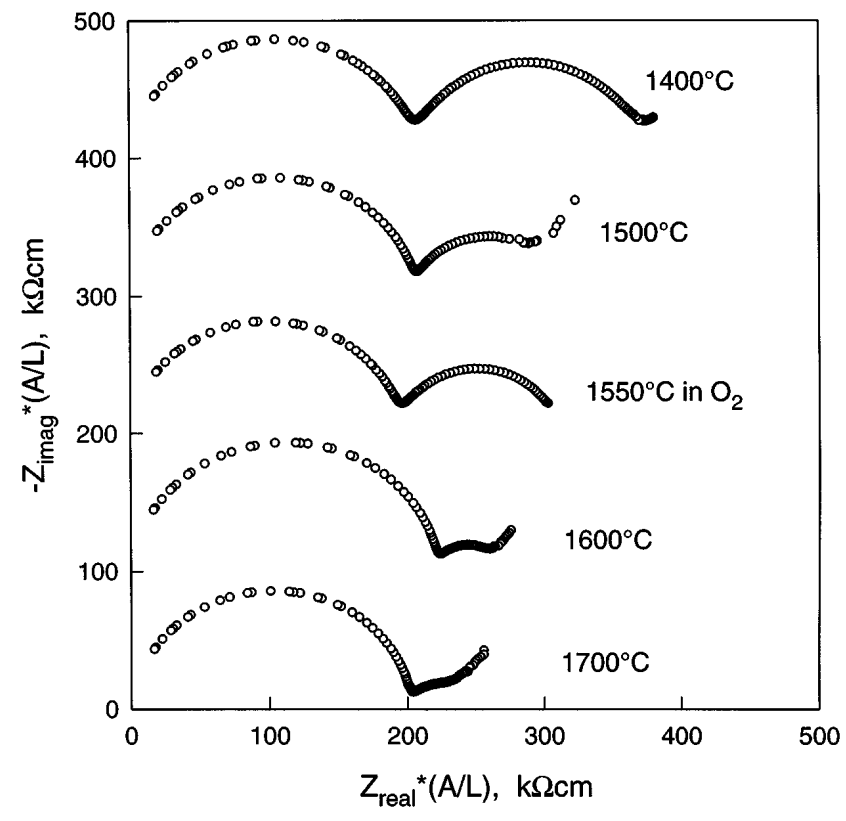

FIG. 14. Nyquist impedance plots obtained at $100{ }^{\circ} \mathrm{C}$ for $\mathrm{BaCe}_{0.85}-$ $\mathrm{Gd}_{0.15} \mathrm{O}_{3-\delta}$ sintered in air (unless otherwise stated) at the temperatures indicated. Atmosphere is $\mathrm{H}_{2} \mathrm{O}$-saturated argon. The absolute impedance has been multiplied by the geometric factor, $A / L$, to permit a direct comparison between samples of slightly different dimensions. Each curve is offset by an amount $100 \mathrm{k} \Omega \mathrm{cm}$ along the imaginary axis.

is approximately $R_{\text {bulk }}$ for subcase (c). In all cases, the resistance of the second $R Q$ circuit, $R_{2}$, is much smaller than that of the first. For the first two subcases, $R_{2}$ is

$$
R_{2}=R_{g b \perp}=2 R_{g b \|}\left(\frac{g}{G}\right)^{2} \approx 2 R_{1}\left(\frac{g}{G}\right)^{2},
$$

whereas it is given by Eq. (9) for the third. The capacitance of the second $R Q$ circuit is, for all subcases, given by

$$
C_{2}=C_{g b \perp}=\frac{1}{2} C_{g b \|}\left(\frac{G}{g}\right)^{2} \approx C_{1}\left(\frac{G}{g}\right),
$$

where the latter approximation is obtained from Eq. (14).

Because of the differences in response of the first and second subcircuits, the Nyquist plot of a material in which $\sigma_{g b}>\sigma_{\text {bulk }}$ would, in principle, exhibit two arcs. The high frequency arc would correspond to the $R_{2} Q_{2}$ subcircuit of grain boundaries in series with (or perpendicular to) grains, and the lower frequency arc to the combined $R_{1} Q_{1}$ subcircuit of grain interiors and parallel grain boundaries, in contrast to what is typically observed. This is a direct consequence of the fact that $R_{2} C_{2}<R_{1} C_{1}$ [as per Eqs. (6), (12), and (13)]. In practice, such a two-arc spectrum is not obtained because the radius of the high frequency are, $R_{2}$, is many orders of magnitude smaller than that of the "low" frequency arc. For example, if $\sigma_{g b} / \sigma_{\text {bulk }} \gg 1 / 2 G / g$ and $g / G \sim$



FIG. 15. The conductivities, $\sigma_{1}$ and $\sigma_{2}$, at $100{ }^{\circ} \mathrm{C}$ for batch-2 samples of $\mathrm{BaCe}_{0.85} \mathrm{Gd}_{0.15} \mathrm{O}_{3-\delta}$ as a function of sintering temperature. Atmosphere is $\mathrm{H}_{2} \mathrm{O}$-saturated argon. Error bars reflect the standard deviations obtained from the equivalent circuit fitting routine, and are smaller than the symbol size in the case of $\sigma_{1}$. Solid lines are guides to the eye.

$10^{-2}$, then $R_{2} / R_{1} \sim 10^{-4}$. Consequently, the Nyquist plot will exhibit a single arc, which, for all practical purposes, intersects the origin. If one knew at the outset that $\sigma_{g b} / \sigma_{\text {bulk }} \gg 1 / 2 G / g$, one could justifiably interpret the $Y$ and $n$ values obtained from this single arc as bulk properties, and, from macroscopic sample dimensions, determine $\epsilon_{\text {bulk }}$. The $R$ obtained from this single arc would be correctly interpreted as a grain boundary property (parallel grain boundaries); however, it could only be used to determine $\sigma_{g b}$ in combination with independent microstructural investigations. In the absence of independent information about the relative values of $\sigma_{g b}$ and $\sigma_{\text {bulk }}$, it would be inappropriate to assume that $R_{1}$ is dominated by either the resistance of parallel grain boundaries or that of grain interiors.

\section{Case (3): $\sigma_{g b} \sim \sigma_{\text {bulk }}$}

The case in which the grain boundary and bulk conductivities of a material with typical microstructure are approximately equal is quite similar to case (1), $\sigma_{\text {bulk }}>\sigma_{g b}$ : Eq. (8) reduces to Eq. (9), and Eq. (6) reduces to Eq. (10). The requirements for the observation of two arcs in the Nyquist plot are also similar to those of case (1): (i) $R_{1}$ and $R_{2}$ should not differ by more than a few orders of magnitude, and (ii) $\omega_{0,1}$ and $\omega_{0,2}$ should differ significantly. The ratio of $R_{2} / R_{1}$ is given by Eq. (9) and will be on the order of $10^{-2}$ or smaller, for typical microstructures. While this value is rather small, it is not implausible that two arcs that differ in radius by a factor of 100 could be resolved in a Nyquist plot. The values of the characteristic frequencies, on the other hand, will 


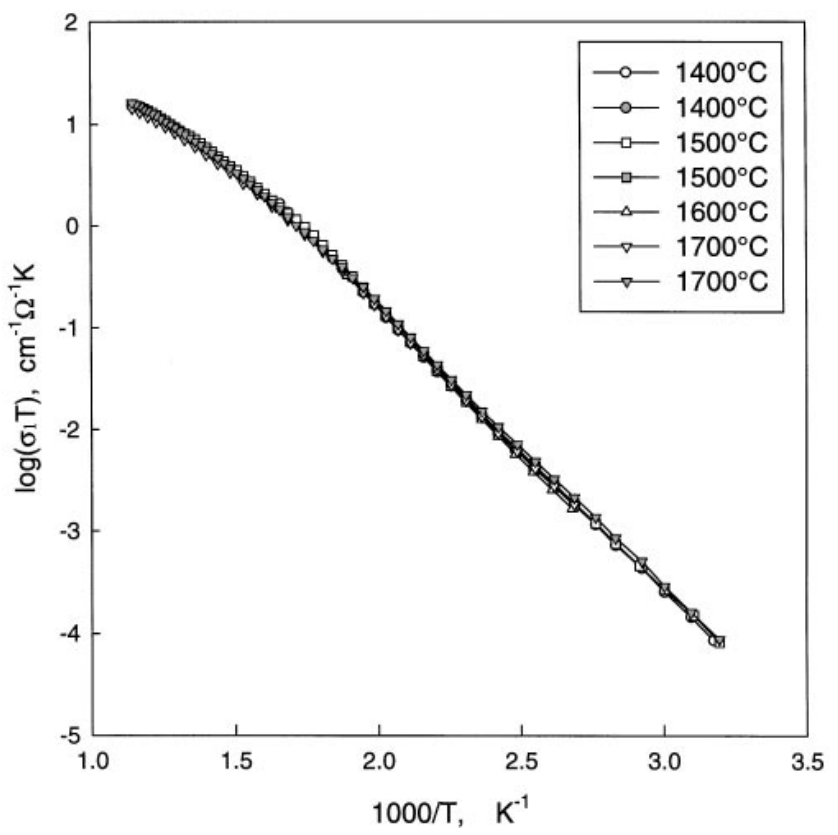

(a)

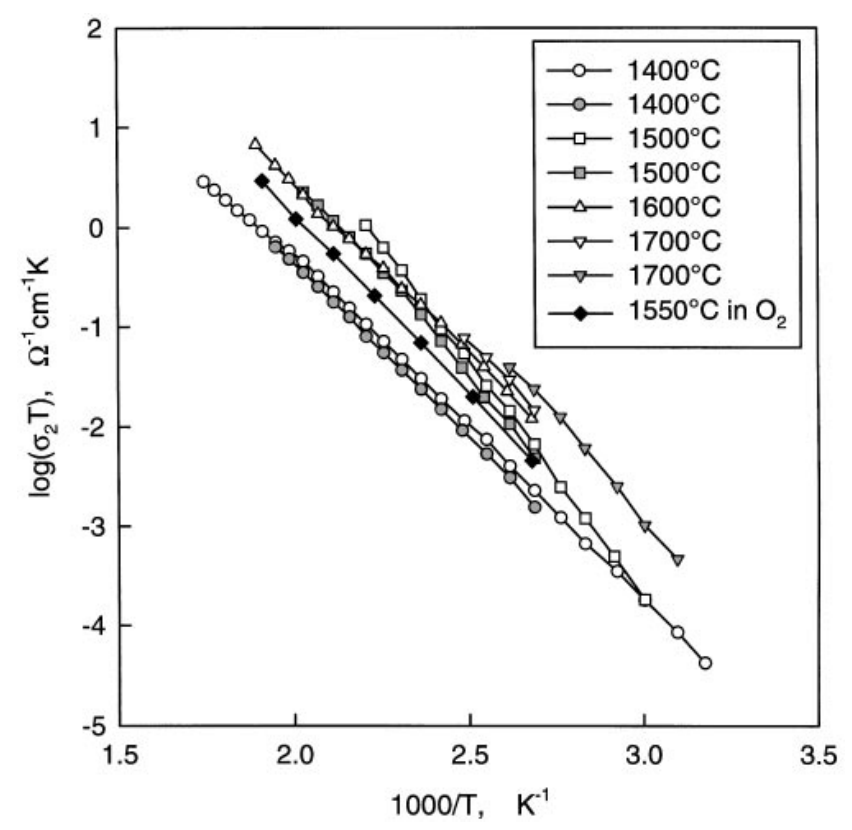

(b)

FIG. 16. The conductivities, (a) $\sigma_{1}$ and (b) $\sigma_{2}$, of $\mathrm{BaCe}_{0.85} \mathrm{Gd}_{0.15^{-}}$ $\mathrm{O}_{3-\delta}$ as a function of temperature plotted in Arrhenius form. Atmosphere is $\mathrm{H}_{2} \mathrm{O}$-saturated argon. In (a) data from batch-1 samples are omitted as these are coincident with data shown. In (b), for clarity, only the data from one representative batch-1 sample are shown.

be virtually identical under the assumption that $\epsilon_{\text {bulk }} \sim$ $\epsilon_{g b}$, and consequently, only one arc will be observed. Moreover, as was pointed out in the discussion of case (1), the conductivity, $\sigma_{1}$, obtained from this single arc will be within $2 \%$ of $\sigma_{\text {bulk }}$ if $\sigma_{g b}$ is indeed comparable in magnitude to $\sigma_{\text {bulk }}$.

\section{Example}

Returning now to the data presented in Fig. 1, the analysis above reveals that only for case (1), $\sigma_{\text {bulk }}>$ $\sigma_{g b}$, can two arcs be observed. Furthermore, Eqs. (10) and (7) are valid for the calculation of the bulk and grain boundary conductivities, respectively. Least squares fitting of the impedance data to the equivalent circuit model of Fig. 2 yielded the parameters: $R_{1}=5200 \Omega, Y_{1}=$ $9.20 \times 10^{-11}, n_{1}=0.905 ; R_{2}=1620 \Omega, Y_{2}=3.48 \times$ $10^{-8}$, and $n_{2}=0.907$. The quality of the fit is shown in Fig. 5. The geometric factor, $A / L$ is $5.64 \mathrm{~cm}$, and the grain size is approximately $3 \times 10^{-4} \mathrm{~cm} .{ }^{15}$ The conductivity of the grain interiors is simply $\sigma_{1}=9.8 \times$ $10^{-6} \Omega^{-1} \mathrm{~cm}^{-1}$. The ratio $C_{1} / C_{2}$ is $1.57 \times 10^{-3}$, which, when combined with the value of $\sigma_{2}$, yields a (specific) grain boundary conductivity of $1.72 \times 10^{-7} \Omega^{-1} \mathrm{~cm}^{-1}$. Multiplying the ratio of the capacitances by the average grain size yields a grain boundary thickness of $\sim 50 \AA$, a reasonable value that justifies the assumptions behind Eq. (14). These results demonstrate that the grain boundaries are, in fact, much more resistive that the grain interiors, despite the apparently small value of $R_{2}$.

\section{Summary}

In summary, it has been experimentally observed that the Nyquist plot obtained from polycrystalline materials exhibits either one or two arcs. Analysis of the brick layer model reveals that there is only case for which two arcs will be observed, that is, for a material with a typical microstructure, i.e., $g / G<10^{-2}$, only when $\sigma_{\text {bulk }}>\sigma_{g b}$. Thus, for a two-arc spectrum, one can immediately conclude that grain boundaries are more resistive than the bulk, regardless of the relative magnitudes of the resistances associated with these arcs. Furthermore, the first (high frequency) arc represents only the bulk response, without any significant parallel grain boundary contribution, and the second (mid to low frequency) arc represents the series grain boundary response. The bulk and grain boundary conductivities are given by $\sigma_{1}$ and $\sigma_{2}(g / G)$, respectively. If the bulk and grain boundary dielectric constants are approximately equal, the grain boundary conductivity is then given by $\sigma_{2}\left(C_{1} / C_{2}\right)$. The observation of two arcs, where $\epsilon_{g b}$ $\epsilon_{\text {bulk }}$, also necessarily implies that $\sigma_{\text {bulk }}>\sigma_{g b}$, as only a difference in resistivities between two regions of the material can be responsible for a difference in characteristic frequencies.

In a Nyquist plot in which only one arc is observed, the impedance data alone will not be sufficient to reveal the magnitudes of either $\sigma_{\text {bulk }}$ or $\sigma_{g b}$. It is possible for the resistance associated with this single arc to correspond to either the bulk resistance (cases $2 \mathrm{c}$ and 3 ), the parallel grain boundary resistance (case 2a), or a combination thereof (case $2 b$ ). 
TABLE II. The electrical properties measured in $\mathrm{H}_{2} \mathrm{O}$-saturated argon of polycrystalline $\mathrm{BaCe}_{0.85} \mathrm{Gd}_{0.15} \mathrm{O}_{3-\delta}$ synthesized under various sintering conditions. The pre-exponential term, $A$, and the activation energy, $E_{\sigma}$, were obtained from a fit of the data to $\sigma=A / T \exp \left(E_{\sigma} / k_{b} T\right)$ over the temperature range $\sim 100-300{ }^{\circ} \mathrm{C}$. The pre-exponential term, $A$, for grain boundaries refers to the total grain boundary conductivity rather than specific. Number in parentheses indicates experimental uncertainty in the final digit.

\begin{tabular}{|c|c|c|c|c|c|c|}
\hline \multirow[b]{2}{*}{ Batch } & \multicolumn{4}{|c|}{$\sigma_{1}$ (bulk) } & \multicolumn{2}{|c|}{$\sigma_{2}$} \\
\hline & sinter $T\left({ }^{\circ} \mathrm{C}\right)$ & sinter atm & $E_{\sigma}(\mathrm{ev})$ & $\log (A)(\mathrm{K} / \Omega \mathrm{cm})$ & $E_{\sigma}(\mathrm{eV})$ & $\log (A)(\mathrm{K} / \Omega \mathrm{cm})$ \\
\hline 1 & 1550 & dry $\mathrm{O}_{2}$ & $0.576(5)$ & $4.97(5)$ & $0.718(5)$ & $7.39(6)$ \\
\hline 1 & 1550 & dry $\mathrm{O}_{2}$ & $0.577(2)$ & $5.03(2)$ & $0.734(3)$ & 7.43(4) \\
\hline 1 & 1550 & dry $\mathrm{O}_{2}$ & $0.547(5)$ & $4.69(6)$ & $0.741(4)$ & $7.49(5)$ \\
\hline 2 & 1400 & air & $0.561(3)$ & $4.87(4)$ & $0.674(4)$ & $6.48(5)$ \\
\hline 2 & 1400 & air & $0.562(4)$ & $4.85(5)$ & $0.696(3)$ & $6.66(4)$ \\
\hline 2 & 1500 & air & $0.560(3)$ & $4.87(3)$ & $0.94(1)$ & $10.4(1)$ \\
\hline 2 & 1500 & air & $0.575(4)$ & $5.02(4)$ & $0.81(1)$ & $8.7(2)$ \\
\hline 2 & 1600 & air & $0.583(3)$ & $5.07(3)$ & $0.675(5)$ & $7.24(6)$ \\
\hline 2 & 1700 & air & $0.562(3)$ & $4.85(3)$ & $0.71(4)$ & $7.8(5)$ \\
\hline 2 & 1700 & air & $0.547(2)$ & $4.75(2)$ & $0.82(2)$ & $9.5(3)$ \\
\hline average & & & $0.56(1)$ & $4.9(1)$ & $\cdots$ & $\ldots$ \\
\hline
\end{tabular}

The brick layer model discussed here assumes an admittedly simplistic microstructure for a polycrystalline material. This approach has been criticized by de Jonghe, in particular, primarily for its inapplicability to anisotropic materials such as $\beta$-alumina. ${ }^{16}$ However, the error introduced by the reduction of a complex microstructure to a uniform array of cube for an isotropic material is not expected to be large enough that a more realistic model would significantly impact the conclusions drawn. Tortuous grain boundary pathways may be longer than direct pathways by, say, a factor of 2 , but the orders of magnitude arguments made here will not be affected. In addition, Nafe has analyzed the conductive behavior of several materials, examining, in particular, the change from bulk conduction mecha-

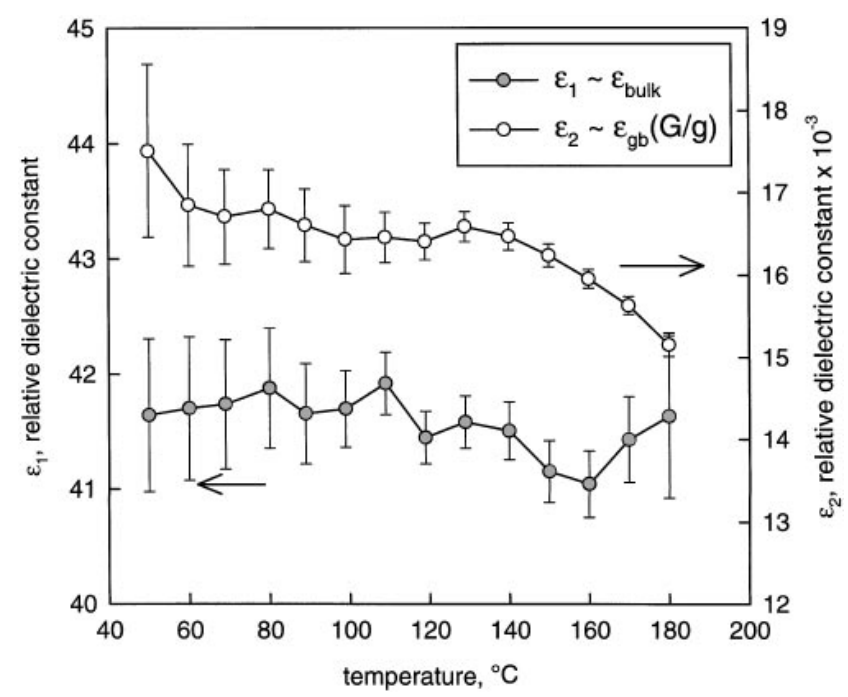

FIG. 17. The relative dielectric constants $\epsilon_{1}$ and $\epsilon_{2}$ for a batch-2 sample of $\mathrm{BaCe}_{0.85} \mathrm{Gd}_{0.15} \mathrm{O}_{3-\delta}$ sintered at $1400{ }^{\circ} \mathrm{C}$. Atmosphere is $\mathrm{H}_{2} \mathrm{O}$-saturated argon. Error bars reflect the standard deviations obtained from the equivalent circuit fitting routine. nisms to grain boundary conduction mechanisms as a function of temperature, and proven the effectiveness of the brick layer model. ${ }^{8}$ This approach has also been applied successfully to doped and undoped $\mathrm{CeO}_{2}$ single crystals and polycrystalline materials ${ }^{10,17}$ and to yttriastabilized zirconia. ${ }^{9,14,18}$ Bonanos et al. ${ }^{7}$ have recently reviewed several microstructural models for the interpretation of impedance spectra, including the "parallel layer" and "series layer" models, ${ }^{19}$ the "constricted pathway" model, ${ }^{20,21}$ and the "effective medium" model. ${ }^{19,22}$ They have shown that the layer models are each a specific case of the brick layer model, while the brick layer model and the effective medium model, in turn, converge for $g / G \rightarrow 0$. The constricted pathway model, while very different in nature from the other models discussed, can only apply to a situation in which (i) there is an ion-blocking secondary phase at the grain boundary and (ii) the activation energies associated with $\sigma_{1}$ and $\sigma_{2}$ are identical. As will be shown below, neither holds for barium cerate. In the present work we provide experimental support for the brick layer model by demonstrating that $\sigma_{2}$ can be varied independently of $\sigma_{1}$, and also show, though these studies, that the bulk conductivity of barium cerate is much greater than that along grain boundaries.

\section{EXPERIMENTAL}

\section{A. Sample preparation}

Crystalline powders of $\mathrm{BaCe}_{0.85} \mathrm{Gd}_{0.15} \mathrm{O}_{3-\delta}$ and $\mathrm{BaCe}_{0.9} \mathrm{Gd}_{0.1} \mathrm{O}_{3-\delta}$ were synthesized by solid-state reaction from high purity $\mathrm{BaCO}_{3}, \mathrm{CeO}_{2}$, and $\mathrm{Gd}_{2} \mathrm{O}_{3}$ following one of two sets of procedures. "Batch-1" powders were ball-milled and subsequently calcined in air at $1250{ }^{\circ} \mathrm{C}$ for $12 \mathrm{~h}$. Pressed pellets were sintered at $1650{ }^{\circ} \mathrm{C}$ for $10-12 \mathrm{~h}$ in dry $\mathrm{O}_{2}$ (gas passed through a $\mathrm{CaSO}_{4}$ drying column) in order to minimize 
TABLE III. The relative bulk and total grain boundary dielectric constants of samples of $\mathrm{BaCe}_{0.85} \mathrm{Gd}_{0.15} \mathrm{O}_{3-\delta}$ prepared under various sintering conditions. In addition, the ratio of the grain size to the grain boundary thickness, $G / g$, the grain size, $G$, as measured from electron micrographs, and the estimated grain thickness, $g$, are also provided. Number in parentheses indicates experimental uncertainty in the final digit.

\begin{tabular}{|c|c|c|c|c|c|c|c|}
\hline Batch & sinter $T\left({ }^{\circ} \mathrm{C}\right)$ & sinter atm & $\epsilon_{1}\left(=\epsilon_{\text {bulk }}\right)$ (unitless) & $\epsilon_{1}\left(=\epsilon_{g b} G / g\right) \times 10^{4}$ & $G / g \times 10^{-2}$ (unitless) & $G(\mu \mathrm{m})$ & $g(\AA)$ \\
\hline 1 & 1550 & dry $\mathrm{O}_{2}$ & $39.8(7)$ & $2.2(7)$ & $6.2(4)$ & \multirow{3}{*}{$\mathrm{n} / \mathrm{a}$} & \multirow{3}{*}{$\mathrm{n} / \mathrm{a}$} \\
\hline 1 & 1550 & dry $\mathrm{O}_{2}$ & $43.2(5)$ & $2.4(5)$ & $5.7(2)$ & & \\
\hline 1 & 1550 & dry $\mathrm{O}_{2}$ & $40(2)$ & $2.5(2)$ & $5.6(2)$ & & \\
\hline 2 & 1400 & air & $36.5(5)$ & $1.4(1)$ & $3.9(2)$ & \multirow{2}{*}{$1.3(2)$} & \multirow{2}{*}{$33(5)$} \\
\hline 2 & 1400 & air & $41.6(2)$ & $1.6(1)$ & $3.9(1)$ & & \\
\hline 2 & 1500 & air & $45.0(5)$ & $4.2(6)$ & $9(2)$ & \multirow{2}{*}{$2.9(3)$} & \multirow{2}{*}{$27(5)$} \\
\hline 2 & 1500 & air & $39.8(4)$ & $5.0(6)$ & $13(2)$ & & \\
\hline 2 & 1600 & air & 43.1(4) & $11.2(8)$ & $26(2)$ & $5.0(6)$ & $19(3)$ \\
\hline 2 & 1700 & air & $43.8(3)$ & $17(2)$ & $39(4)$ & \multirow[t]{2}{*}{ 11(1) } & \multirow[t]{2}{*}{$30(4)$} \\
\hline 2 & 1700 & air & $49.1(2)$ & $16(1)$ & $33(2)$ & & \\
\hline
\end{tabular}

Average bulk dielectric constant $=42(3)$; average grain boundary thickness $=27(6) \AA$.

the dissolution of $\mathrm{H}_{2} \mathrm{O}$ into the material during the processing steps. Both 10 and $15 \%$ Gd-doped barium cerate was prepared in this manner. Processing of "batch2" samples (of composition $\mathrm{BaCe}_{0.85} \mathrm{Gd}_{0.15} \mathrm{O}_{3-\delta}$ ) differed in that calcination was carried out at $1100{ }^{\circ} \mathrm{C}$ for $8 \mathrm{~h}$ and pellets were sintered in stagnant air for $4 \mathrm{~h}$. Moreover, four sets of samples, sintered at 1400, 1500, 1600, and $1700{ }^{\circ} \mathrm{C}$, respectively, were prepared.

$\mathrm{X}$-ray powder diffraction patterns were collected both after calcination and after sintering (dense pellets were ground into a fine powder for this purpose) to confirm that single phase barium cerate had been obtained. In some cases, loss of barium oxide during sintering resulted in the formation of $\mathrm{CeO}_{2}$ on the surface of sintered pellets, and this was removed by polishing prior to collecting diffraction or electrical data. The densities of sintered pellets were determined from a simple measurement of sample dimensions and weight. Density determination by the Archemides method was prevented by the tendency of pellets to disintegrate when placed in various liquids.

The growth and compositional characterization of single crystals with nominal stoichiometry $\mathrm{BaCe}_{0.85^{-}}$ $\mathrm{Gd}_{0.15} \mathrm{O}_{3-\delta}$ and $\mathrm{BaCe}_{0.9} \mathrm{Gd}_{0.1} \mathrm{O}_{3-\delta}$ have been reported elsewhere. ${ }^{23}$ Single-crystal fibers, $\sim 500 \mu \mathrm{m}$ in diameter, were obtained by laser-heated pedestal growth, a variation of the float-zone method. ${ }^{24}$ Dense polycrystalline rods, prepared from batch-1 powders, served as the feed material. Resultant fibers were examined by both single-crystal (Crystal Logic 4-circle diffractometer) and powder x-ray diffraction.

\section{B. Microstructural characterization and chemical analysis}

Optical photographs, showing differences in coloration of batch-2 samples, were recorded with a $35 \mathrm{~mm}$ camera prior to electrical characterization. The grain sizes and compositions of these samples were meas- ured using electron microscopy and microprobe techniques, respectively. Pellets were mounted into an epoxy resin, cut, polished, and etched with concentrated HF. Backscatter micrographs of carbon-coated samples were recorded with a JEOL JXA-733 SEM. The intensities of characteristic X-radiation peaks were collected with five wavelength dispersive spectrometers attached to this microscope. Intensities were converted to stoichiometric quantities using the CITZAF correction program. ${ }^{25}$ The compounds $\mathrm{CePO}_{4}, \mathrm{GdPO}_{4}$, and $\mathrm{BaTiSi}_{3} \mathrm{O}_{9}$ served as standards. The grain boundary regions of batch-1 samples were examined with a Philips 430 transmission electron microscope, and preliminary results of these studies have been reported previously. ${ }^{23}$ Thin foils were prepared by ion milling at room temperature and subsequently carbon-coated prior to TEM characterization. Diffraction contrast imaging was employed to detect any minor phases present at grain boundaries. Compositional analysis of these pellets and of single-crystals was carried out using a JEOL 5200 SEM equipped with an energy dispersive spectrometer (EDS), as also reported earlier. ${ }^{23}$ Neither standards nor an adequate means of deconvoluting overlapping peaks were available at the time of the data collection, and only the ratios of characteristic peak intensities were recorded for these samples.

\section{AC impedance spectroscopy}

For both batch-1 and batch-2 samples, impedance data were collected with an HP 4284A precision LCR (inductance-capacitance-reisistance) meter, over the frequency range $\sim 20 \mathrm{~Hz}$ to $1 \mathrm{MHz}$. The electrodes consisted of Pt that was sputter-coated onto the surfaces of polished pellets. After the application of the electrodes, batch-1 pellets and single crystals were annealed in flowing, dry argon at $500{ }^{\circ} \mathrm{C}$ for $30-60$ min prior to data collection in order to obtain good electrode contact. Impedance spectra were collected in dry $\mathrm{H}_{2} \mathrm{O}$-saturated, and $\mathrm{D}_{2} \mathrm{O}$-saturated argon upon cooling from $500{ }^{\circ} \mathrm{C}$ to 

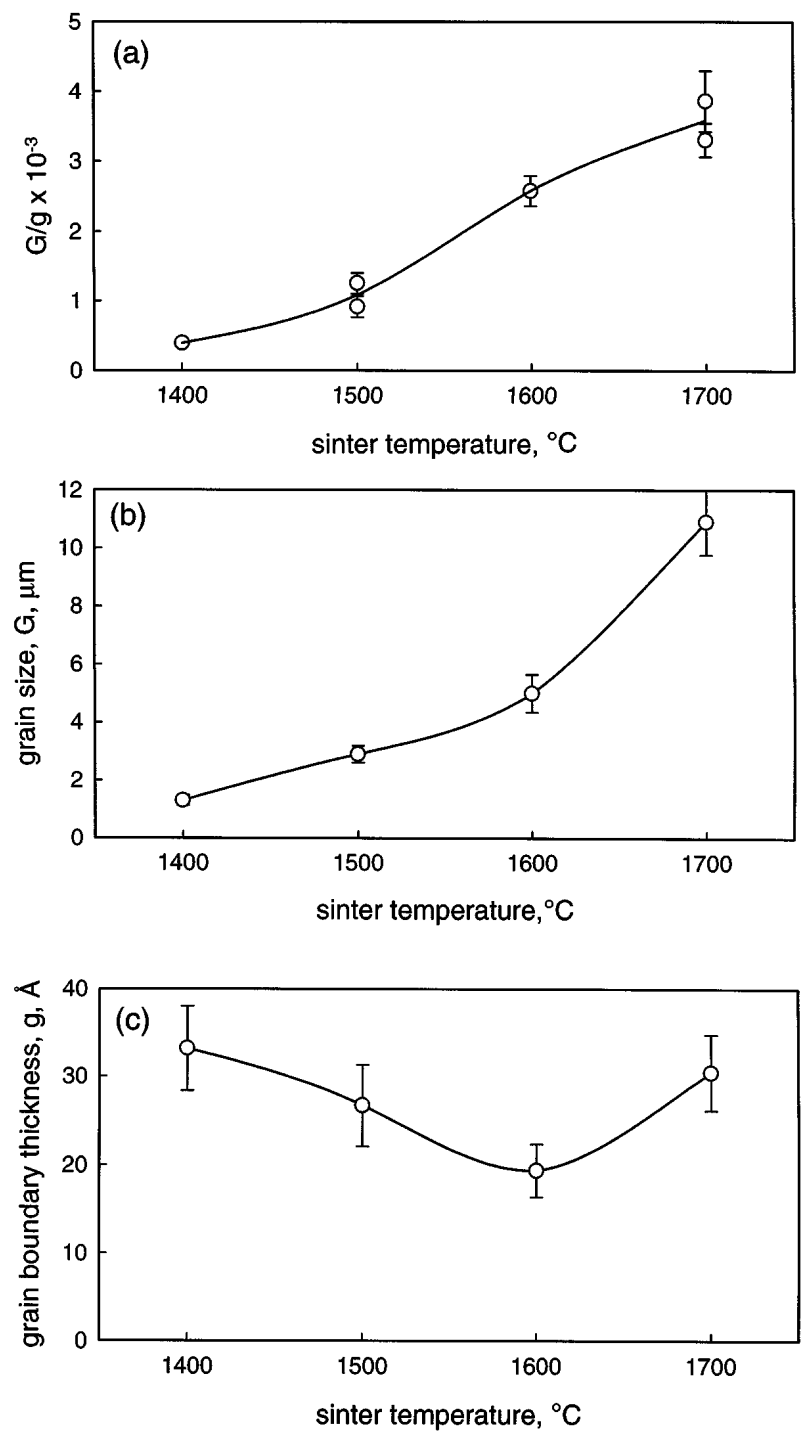

FIG. 18. Parameters describing the microstructure of batch-2 samples of $\mathrm{BaCe}_{0.85} \mathrm{Gd}_{0.15} \mathrm{O}_{3-\delta}$ as a function of sintering temperature: (a) $G / g$, the ratio of the grain diameter to the grain boundary thickness, as determined from a ratio of the dielectric constants; (b) $G$, the grain diameter, as determined from direct microstructural observations; and (c) $g$, the grain boundary thickness as determined from (a) and (b). Solid lines are guides to the eye.

$\sim 100{ }^{\circ} \mathrm{C}$ in $25{ }^{\circ} \mathrm{C}$ increments. Absence of water in the dry argon measurements was ensured by passing the gas through a $\mathrm{CaSO}_{4}$ sieve. Initial equilibration with $\mathrm{H}_{2} \mathrm{O}$ or $\mathrm{D}_{2} \mathrm{O}$ containing atmospheres was conducted at $400{ }^{\circ} \mathrm{C}$ for $12 \mathrm{~h}$. During conductivity measurement, samples were equilibrated for $10-15 \mathrm{~min}$ at each temperature before data were collected. The applied ac voltage was $0.9 \mathrm{~V}$. Impedance data were fit to an equivalent circuit model using the least squares refinement program EQUIVCRT. ${ }^{25}$

Sample preparation and data collection procedures for batch-2 pellets were similar with the following ex-



FIG. 19. The true or specific grain boundary conductivity $\sigma_{g b}$, of $\mathrm{BaCe}_{0.85} \mathrm{Gd}_{0.15} \mathrm{O}_{3-\delta}$ at $100{ }^{\circ} \mathrm{C}$ as a function of sintering temperature. Atmosphere is $\mathrm{H}_{2} \mathrm{O}$-saturated argon. Filled symbol represents data from samples sintered in oxygen (batch-1); all others are sintered in air (batch-2). Error bars reflect the uncertainty in the measurement of both $\sigma_{2}$ and $G / g$.

ceptions: platinum electrodes were annealed at $700{ }^{\circ} \mathrm{C}$ for $1 \mathrm{~h}$, data were collected only in $\mathrm{H}_{2} \mathrm{O}$-saturated atmospheres, and saturation was carried out at $600{ }^{\circ} \mathrm{C}$ for $12 \mathrm{~h}$. Impedance spectra were measured every $10-20{ }^{\circ} \mathrm{C}$ steps (also upon cooling) from 600 to $\sim 100{ }^{\circ} \mathrm{C}$, after an equilibration time of $\sim 55 \mathrm{~min}$ at each temperature. The applied ac voltage was either 50 or $100 \mathrm{mV}$.

\section{RESULTS}

\section{A. Physical characterization}

The x-ray powder diffraction samples of the calcined materials confirmed that essentially single phase barium cerate had been obtained. Calcined powder of the batch1 material with a $15 \%$ Gd dopant level contained trace amounts of $\mathrm{BaGd}_{2} \mathrm{O}_{4}$, less than 1 wt. \%, as evidenced by the presence of two very weak peaks at 29.4 and $30.5^{\circ}$ $2 \theta$ [Fig. 6(a)]. The batch-2 powders contained trace, again, less than 1 wt. \%, amounts of unreacted $\mathrm{BaCO}_{3}$ as evidenced, in this case, by the presence of a very weak peak at $23.5^{\circ} 2 \theta$ [Fig. 6(b)]. X-ray powder diffraction patterns collected after sintering showed no evidence of either of these phases in any sample (Fig. 7). Using the Burnham routine as implemented in the software program Micro-Cellref from MDI, ${ }^{27}$ pseudo-tetragonal lattice constants were refined from the data obtained from sintered pellets. (An internal $2 \theta$ standard was not employed.) Although barium cerate has an orthorhombic unit cell, ${ }^{28}$ overlap of $h k l$ and $k h l$ peaks prevented accurate determination of all three independent parameters. The results for $\mathrm{BaCe}_{0.9} \mathrm{Gd}_{0.1} \mathrm{CeO}_{3-\delta}$ were $a=6.225(3)$, $c=8.776(6) \AA$, and $V_{u c}=340.1(3) \AA^{3}$; and those for $\mathrm{BaCe}_{0.85} \mathrm{Gd}_{0.15} \mathrm{O}_{3-\delta}$ were $a=6.233(3), c=8.772(6) \AA$, and $V_{u c}=340.8(3) \AA^{3}$. These are within a few standard 


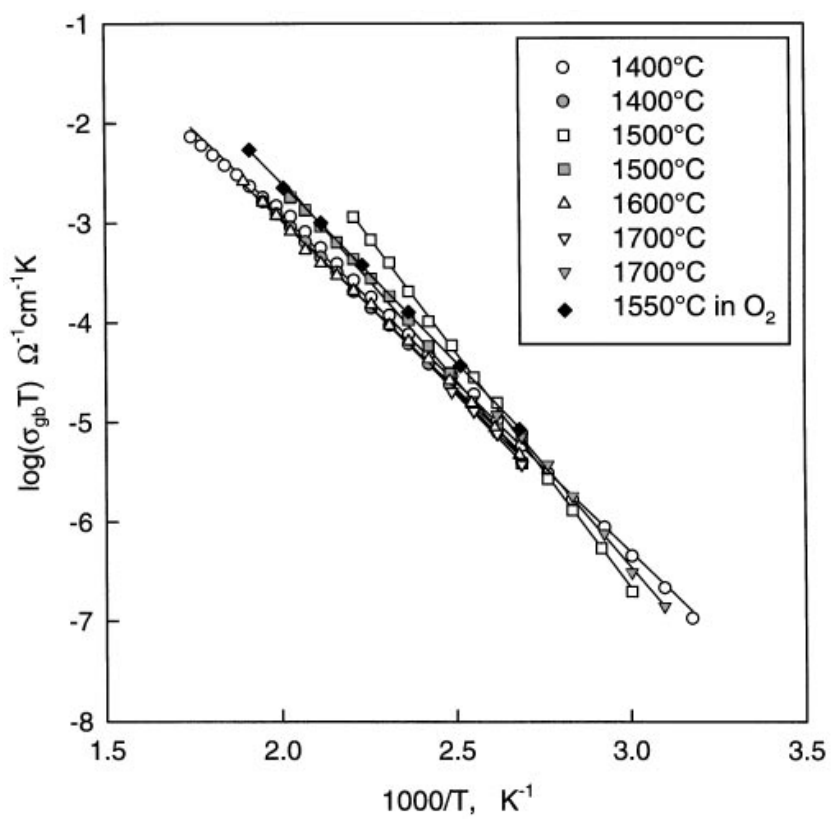

FIG. 20. The specific grain boundary conductivity $\sigma_{g b}$, of one representative batch- 1 and all batch-2 samples of $\mathrm{BaCe}_{0.85} \mathrm{Gd}_{0.15} \mathrm{O}_{3-\delta}$ as a function of temperature, plotted in Arrhenius form. Atmosphere is $\mathrm{H}_{2} \mathrm{O}$-saturated argon. Solid lines show linear regression fits to the data.

deviations of the expected values of $a=6.222(1), b=$ 6.245(1), $c=8.773(2) \AA$, and $V_{u c}=340.9(1) \AA^{3}$ for $\mathrm{BaCe}_{0.9} \mathrm{Gd}_{0.1} \mathrm{CeO}_{2.95}{ }^{28}$

Diffraction peaks, as measured by 4-circle x-ray diffractometry, of fibers obtained from the float-zone LHPG method were rather broad, indicating that the crystals contained a high density of structural defects. Nevertheless, strong peaks could be indexed to a cubic unit cell with $a=4.40(2) \AA$ and $a=4.39$ (4) $\AA$ for the 10 and $15 \%$ doped samples, respectively. Moreover, it was established that growth occurred along the pseudo-cubic [111] direction. Powder diffraction techniques showed that the fibers of nominal composition $\mathrm{BaCe}_{0.85} \mathrm{Gd}_{0.15} \mathrm{O}_{3-\delta}$ contained small precipitates of $\mathrm{CeO}_{2}$, and that these constituted 5-10 wt. \% of the sample, Fig. 8. From the width of the diffraction peaks, the diameter of these inclusions was estimated at $30-35 \mathrm{~nm}$. The pseudo-tetragonal lattice parameters as refined from the powder diffraction data (using $\alpha$-quartz as an internal $2 \theta$ standard) were $a=6.22(3)$ and $c=$ 8.78(4) $\AA$. The presence of $\mathrm{CeO}_{2}$ can be accounted for by the loss of barium oxide during the growth process. The small unit cell volume, $V_{u c}=339(2) \AA^{3}$, is also consistent with a barium deficiency. ${ }^{4}$ In our earlier, preliminary report on the growth and characterization of doped barium cerate single crystals, we had incorrectly concluded, on the basis of the EDS results, that the fibers were of the same composition as the feed material. While differences in the relative intensities of the elemental characteristic lines were noted, these were attributed to

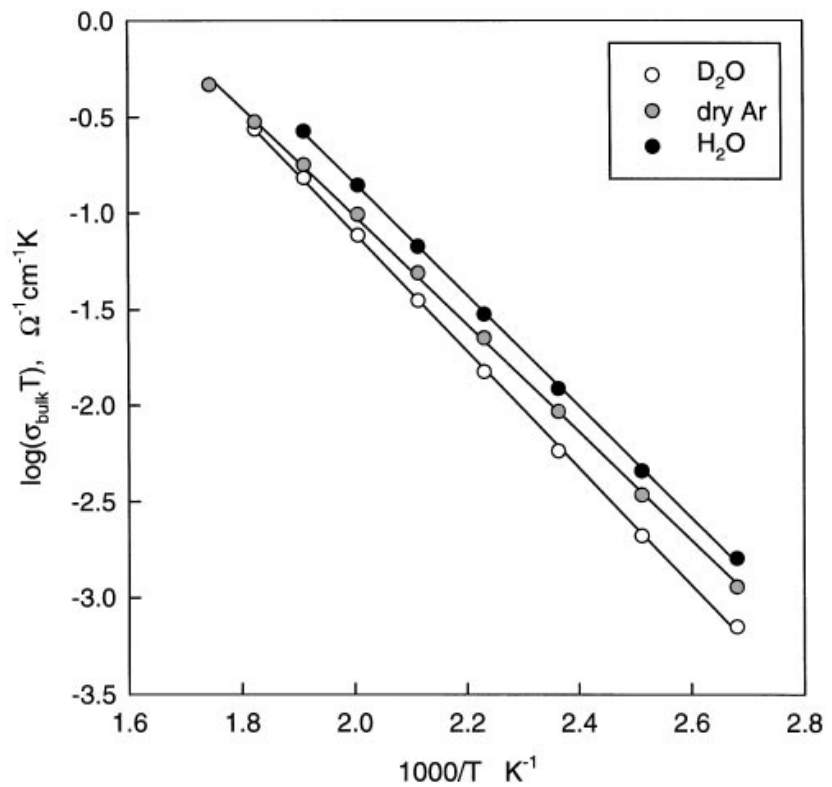

(a)

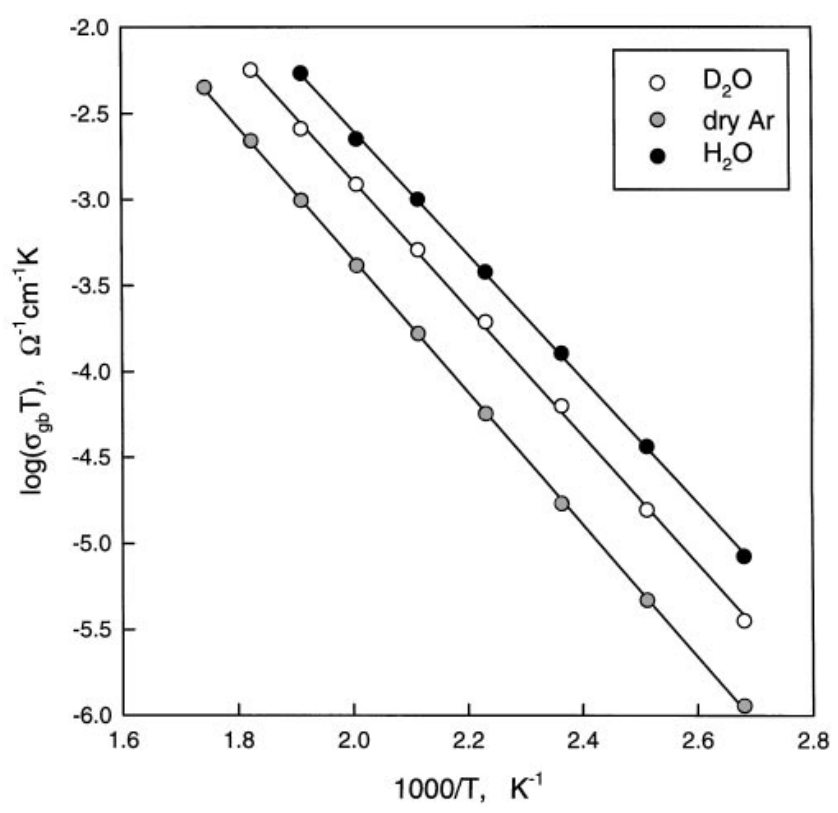

(b)

FIG. 21. The conductivities (a) $\sigma_{1}$ and (b) $\sigma_{2}$ of a batch-1 sample of $\mathrm{BaCe}_{0.85} \mathrm{Gd}_{0.15} \mathrm{O}_{3-\delta}$ as function of temperature in dry, $\mathrm{H}_{2} \mathrm{O}$ - and $\mathrm{D}_{2} \mathrm{O}$-saturated argon. Data are plotted in Arrhenius form. Solid lines show linear regression fits to the data. For clarity, only the low temperature (linear) portion of the data are shown.

statistical variations. For the polycrystalline feed rod, the ratio of the intensity of the $\mathrm{Gd} \mathrm{L}_{\alpha}$ peak to that of the $\mathrm{Ba} \mathrm{L}_{\alpha}$ peak was 0.19(1), and that of the $\mathrm{Ce}_{\beta}$ peak to that of the $\mathrm{Ba} \mathrm{L}_{\alpha}$ peak was $0.21(1)$. The corresponding values for the fiber were $0.22(3)$ and $0.23(3) .{ }^{23}$

Compositional analysis of batch-2 pellets indicated that, within instrumental error, the desired composition had been obtained (Table I). Moreover, there was 
TABLE IV. The electrical properties of polycrystalline $\mathrm{BaCe}_{0.85} \mathrm{Gd}_{0.15} \mathrm{O}_{3-\delta}$ as measured in different atmospheres and for batch-1 samples. The pre-exponential term, $A$, and the activation energy, $E_{\sigma}$, were obtained from a fit of the data to $\sigma=A / T \exp \left(E_{\sigma} / k_{b} T\right)$ over the temperature range $\sim 100-300{ }^{\circ} \mathrm{C}$. The value of $G / g$ determined for the conversion from $\sigma_{2}$ (total) to $\sigma_{g b}$ (specific) for the sample examined in all three atmospheres is also given. In general, the value of this parameter ranged from $5.4 \times 10^{2}$ to $6.2 \times 10^{2}$. Number in parentheses indicates experimental uncertainty in the final digit.

\begin{tabular}{|c|c|c|c|c|c|c|}
\hline \multicolumn{4}{|c|}{ Bulk } & \multicolumn{2}{|c|}{ Grain boundaries } & \multirow[b]{2}{*}{$G / g \times 10^{-2}($ sample 1$)$} \\
\hline atm & $E_{\sigma}(\mathrm{eV})$ & $\log (A)(\mathrm{K} / \Omega \mathrm{cm})$ & $\epsilon_{\text {bulk }}$ & $E_{\sigma}(\mathrm{eV})$ & $\log (A)(\mathrm{K} / \Omega \mathrm{cm})$ & \\
\hline Dry $^{\mathrm{a}}$ & $0.568(4)$ & $4.80(8)$ & $41(2)$ & $0.75(1)$ & $4.36(1)$ & $5.8(2)$ \\
\hline $\mathrm{H}_{2} \mathrm{O}^{\mathrm{b}}$ & $0.56(2)$ & $4.9(2)$ & $41(2)$ & $0.73(1)$ & $4.68(4)$ & $5.6(2)$ \\
\hline $\mathrm{D}_{2} \mathrm{O}^{\mathrm{c}}$ & $0.606(6)$ & 5.01(7) & $40(1)$ & $0.740(9)$ & $4.58(10)$ & $5.4(5)$ \\
\hline
\end{tabular}

${ }^{a}$ As averaged from two samples.

${ }^{\mathrm{b}}$ As averaged from three samples.

${ }^{\mathrm{c}}$ Results from one sample.

no indication of a difference in composition between grain interior and grain boundary regions. Unlike the composition, both the color and the density of sintered pellets varied in a fairly reproducible manner with sintering temperature. Higher sintering temperatures led to a darkening of the greenish color, and at the highest sintering temperature the appearance of a thin yellow layer of $\mathrm{CeO}_{2}$. The change in color likely reflects the increased concentration of $\mathrm{Ce}^{3+}$ ions. In contrast, batch-1 samples, sintered in $\mathrm{O}_{2}$ at $1550{ }^{\circ} \mathrm{C}$, were a pale yellowgreen. While the grain size of batch-2 materials increased monotonically with sintering temperature, Fig. 9 (see also Table III above), the density first increased then decreased at the highest temperature examined (Fig. 10). The drop in density at high sintering temperatures apparently results from grain boundary break-away from pores (see Ref. 29 for a general discussion of densification in ceramic materials); compare Figs. 9(c) and 9(d). Transmission electron micrographs of $\mathrm{BaCe}_{0.9} \mathrm{Gdd}_{0.1} \mathrm{O}_{3-\delta}$ and $\mathrm{BaCe}_{0.85} \mathrm{Gd}_{0.15} \mathrm{O}_{3-\delta}$ (batch-1 samples) are presented in Fig. 11. These micrographs, which are representative of the overall microstructure, demonstrate that the grain boundaries are free of amorphous phases.

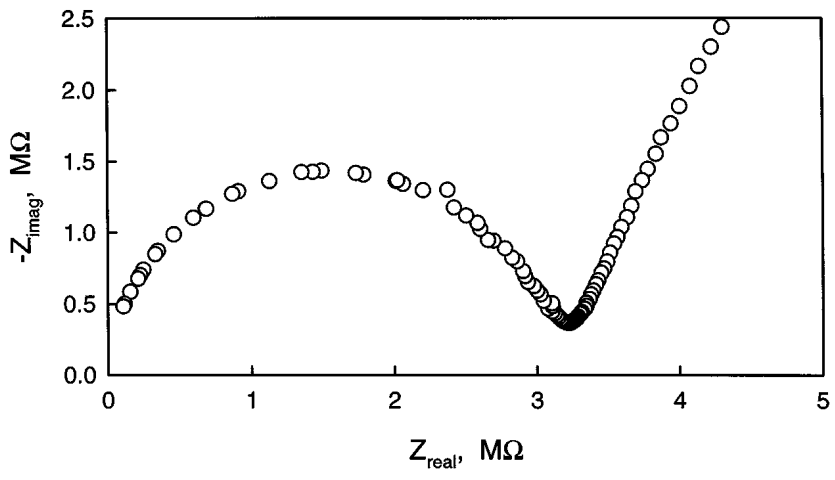

FIG. 22. Nyquist impedance plot obtained at $150{ }^{\circ} \mathrm{C}$ for a singlecrystal fiber of nominal composition $\mathrm{BaCe}_{0.85} \mathrm{Gd}_{0.15} \mathrm{O}_{3-\delta}$. Atmosphere is $\mathrm{H}_{2} \mathrm{O}$-saturated argon.

\section{B. Electrical characterization}

The raw Nyquist plots obtained at selected temperatures from two representative batch-2 samples sintered at 1400 and $1700{ }^{\circ} \mathrm{C}$ are shown in Figs. 12 and 13, respectively. A large arc due to series grain boundaries is apparent in the low temperature Nyquist plots of Fig. 12. At temperatures of $\sim 300{ }^{\circ} \mathrm{C}$ and higher, the series grain boundary impedance is significant; however, the characteristic frequency of even the lower frequency arc (which increases exponentially with temperature) could not be accessed. As a consequence, only the sum of $R_{1}$ and $R_{2}$ could be measured at elevated temperatures,

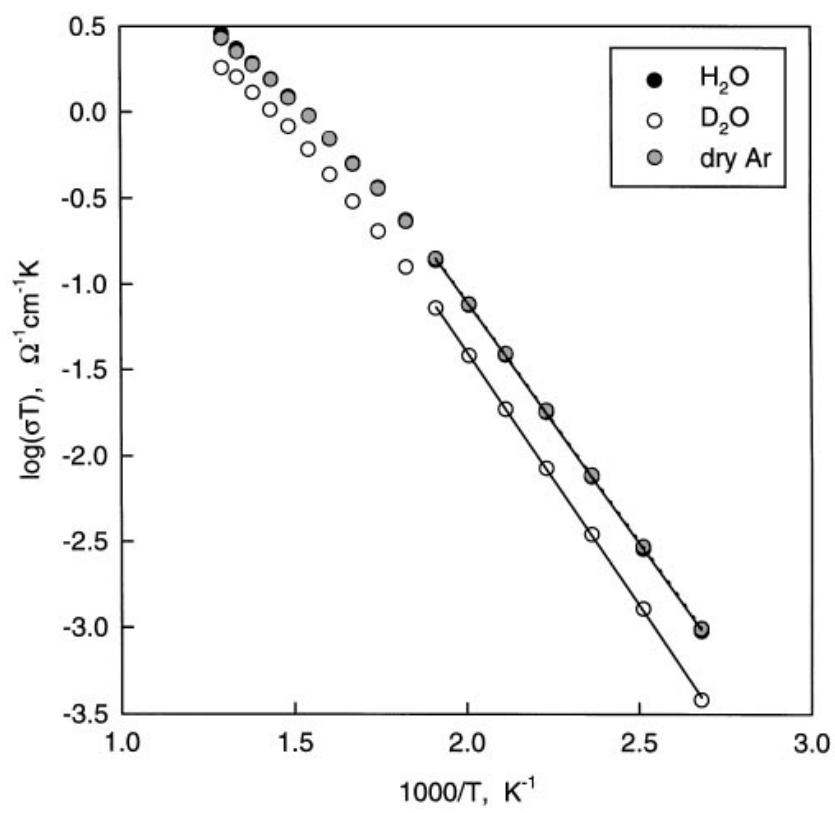

FIG. 23. The conductivity of a single-crystal fiber of nominal composition $\mathrm{BaCe}_{0.85} \mathrm{Gd}_{0.15} \mathrm{O}_{3-\delta}$ as a function of temperature in dry, $\mathrm{H}_{2} \mathrm{O}$ and $\mathrm{D}_{2} \mathrm{O}$-saturated argon. Data are plotted in Arrhenius form. Solid lines show linear regression fits to the low temperature portion of the data. Data in dry argon (gray-filled circles and dotted line) are almost coincident with those in $\mathrm{H}_{2} \mathrm{O}$ saturated argon (black circles and solid line). 
TABLE V. The electrical properties of barium cerate fibers with nominal composition $\mathrm{BaCe}_{0.85} \mathrm{Gd}_{0.15} \mathrm{O}_{3-\delta}$ as measured in different atmospheres and compared to those of polycrystalline $\mathrm{Ba}_{0.96} \mathrm{Ce}_{0.85} \mathrm{Gd}_{0.15} \mathrm{O}_{3-\delta} .{ }^{4}$ The pre-exponential term, $A$, and the activation energy, $E_{\sigma}$, were obtained from a fit of the data to $\sigma=A / T \exp \left(E_{\sigma} / k_{b} T\right)$ over the temperature range $150-250{ }^{\circ} \mathrm{C}$. Data have been averaged for two samples. Number in parentheses indicates experimental uncertainty in the final digit.

\begin{tabular}{lll}
\hline \hline \multicolumn{1}{c}{ atm } & $E_{\sigma}(\mathrm{eV})$ & $\log (A)(\mathrm{K} / \Omega \mathrm{cm})$ \\
\hline Dry & $0.555(4)$ & $4.4(1)$ \\
$\mathrm{H}_{2} \mathrm{O}$ & $0.546(9)$ & $4.3(3)$ \\
$\mathrm{H}_{2} \mathrm{O}, \mathrm{Ba}=0.96$ & $0.563(2)$ & $4.59(2)$ \\
$\mathrm{D}_{2} \mathrm{O}$ & $0.573(7)$ & $4.2(3)$ \\
\hline \hline
\end{tabular}

rather than each term individually. This sum yields the "total" conductivity of the sample, where $1 / \sigma_{\text {total }}=$ $1 / \sigma_{1}+1 / \sigma_{2}$. As will be demonstrated below, the activation energy for grain boundary conductivity is much greater than that of bulk conductivity, and, somewhat fortuitously, at higher temperatures $R_{1}+R_{2} \sim R_{1}$, and hence $\sigma_{\text {total }} \sim \sigma_{1}$. In the case of the sample represented in Fig. 13, the much smaller series grain boundary impedance became negligible by $250{ }^{\circ} \mathrm{C}$, and $\sigma_{1}$ and $\sigma_{\text {total }}$ were indistinguishable above this temperature.

In Fig. 14 the Nyquist plots obtained at $100{ }^{\circ} \mathrm{C}$ for five samples, each processed under the different conditions described above, are shown. The conductivities $\sigma_{1}$ and $\sigma_{2}$ obtained from the Nyquist plots of the batch-2 samples are plotted in Fig. 15, as a function of sintering temperature. The Arrhenius curves, $\log (\sigma T)$ vs $1 / T$, for all processing conditions are presented in Fig. 16, and the corresponding activation energies and pre-exponential terms are given in Table II.

These results suggest, at first glance, that there is a decrease in series grain boundary resistance with increasing sintering temperature, and that sintering in oxygen leads to higher grain boundary resistance than sintering in air. Before converting $\sigma_{2}$ to $\sigma_{g b}$, to permit a comparison of the true grain boundary conductivities, we first note that $\sigma_{1}$ is essentially independent of $\sigma_{2}$. While the latter increases monotonically and substantially with sintering temperature, $\sigma_{1}$ is virtually constant. Thus, the influence of grain boundary conductivity on $\sigma_{1}$ is negligible, in agreement with the predictions of the brick layer model. These results further demonstrate that it is not possible for the conduction process in barium cerate (essentially reflected by $\sigma_{1}$ ) to be dominated by a "fast grain boundary process" (reflected by $\sigma_{2}$ ). A second experimentally important feature of the data presented in Fig. 14 is that because the size of the grain boundary arc increased with decreasing sintering temperature, the grain boundary properties could be determined most accurately for samples sintered at $1400{ }^{\circ} \mathrm{C}$. Samples sintered at $1500{ }^{\circ} \mathrm{C}$, which might also have been expected to yield grain boundary properties to high accuracy,

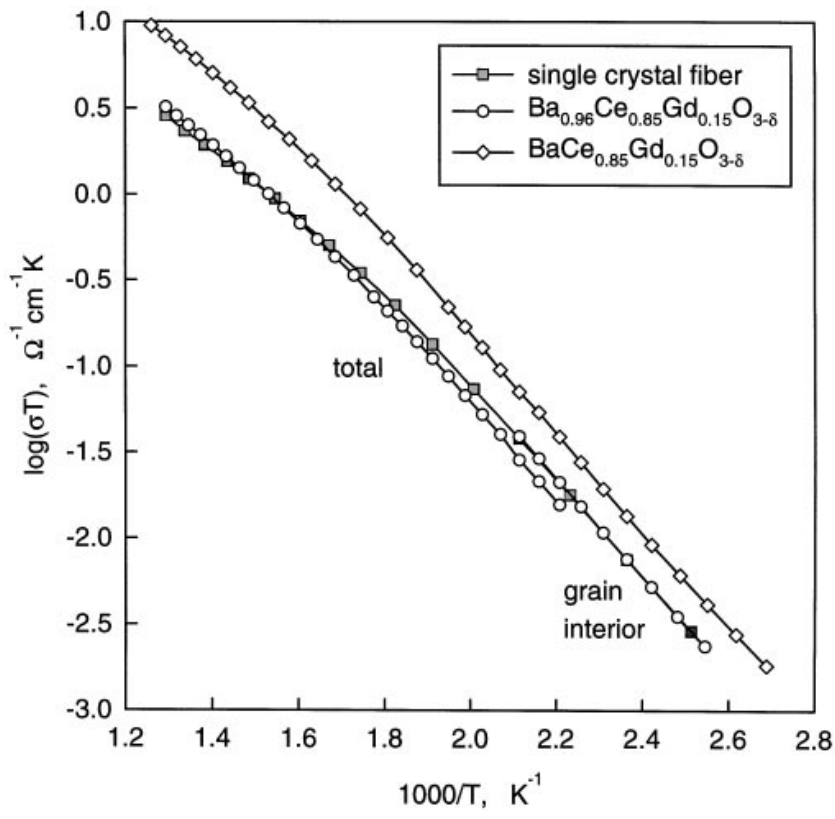

FIG. 24. The conductivity of a single-crystal fiber of nominal composition $\mathrm{BaCe}_{0.85} \mathrm{Gd}_{0.15} \mathrm{O}_{3-\delta}$ as compared to that of polycrystalline $\mathrm{BaCe}_{0.85} \mathrm{Gd}_{0.15} \mathrm{O}_{3-\delta}$ and $\mathrm{Ba}_{0.96} \mathrm{Ce}_{0.85} \mathrm{Gd}_{0.15} \mathrm{O}_{3-\delta} .{ }^{4}$ In the case of the polycrystalline, barium-deficient material, $R_{2}$ remained significant even at temperatures where only the sum $R_{1}$ and $R_{2}$ could be measured; hence both bulk conductivity at low temperatures and total conductivity at high temperatures are presented.

exhibited inexplicably large electrode impedances. As a result, the electrode response obscured the series grain boundary arc (the effect was more significant at higher temperatures than that of Fig. 14) and prevented precise determination of $\sigma_{2}$.

The capacitances associated with both the bulk and series grain boundary responses were determined according to Eq. (8), from which, in turn, $\epsilon_{1} \sim \epsilon_{\text {bulk }}$, $\epsilon_{2} \sim \epsilon_{g b}(G / g)$, and $(G / g)$ were calculated. The results, over the temperature range $50-180{ }^{\circ} \mathrm{C}$ for a batch-2 sample sintered at $1400{ }^{\circ} \mathrm{C}$, are plotted in Fig. 17. (It is only over this somewhat narrow temperature range that a large series grain boundary arc and a significant portion of the bulk arc were obtained, and hence that both $\epsilon_{1}$ and $\epsilon_{2}$ could be reliably measured; see Fig. 12.) The figure reveals that barium cerate has a (bulk) relative dielectric constant of approximately 41 and that it is independent of temperature, as would be expected. Furthermore, the grain boundary dielectric response has the same temperature dependence as that of the bulk, i.e., both are approximately independent of temperature, suggesting that the assumption $\epsilon_{g b} \sim \epsilon_{\text {bulk }}$ is valid. The microstructural parameter, $G / g$, is $3.9(2) \times 10^{2}$ for this sample.

Carrying out the same calculation for all samples yielded the dielectric constants and microstructural parameters listed in Table III. These results are also presented graphically in Fig. 18 for the batch-2 materials. It 
TABLE VI. The values of two parameters quantifying the isotope effect, as measured in single crystal and polycrystalline Gd-doped barium cerate. Number in parentheses indicates experimental uncertainty in the final digit.

\begin{tabular}{lllll}
\hline \multicolumn{1}{c}{ Property } & Crystal $^{\mathrm{a}}$ & gr. int. $^{\mathrm{b}}$ & gr. bnd. $^{\mathrm{b}}$ & Model \\
\hline$A\left(\mathrm{H}_{2} \mathrm{O}\right) / A\left(\mathrm{D}_{2} \mathrm{O}\right)$ & $1.05(5)$ & $0.9(1)$ & $1.1(2)$ & 1.41 \\
$E_{\sigma}\left(\mathrm{H}_{2} \mathrm{O}\right)-E_{\sigma}\left(\mathrm{D}_{2} \mathrm{O}\right), \mathrm{eV}$ & $0.027(3)$ & $0.030(6)$ & $0.022(8)$ & $0.02-0.06$ \\
\hline \hline
\end{tabular}

${ }^{\mathrm{a}}$ As averaged from two samples.

${ }^{\mathrm{b}}$ Results of one sample.

can be seen that the rate of increase of $G / g$ with sintering temperature is almost identical to that of the increase of $G$ as measured directly from electron micrographs. The grain boundary thickness, $g$ estimated simply from the quotient of $G_{\text {microstructural }}$ and $(G / g)_{\text {dielectric }}$, is approximately $30 \AA$ [Fig. 18(c)]. This is an entirely reasonable value, corresponding to about 6 or 7 pseudo-cubic unit cells.

The specific grain boundary conductivity at $100{ }^{\circ} \mathrm{C}$ of each sample is plotted as a function of sintering temperature in Fig. 19, and over the entire temperature range examined in Fig. 20. It is apparent that despite the large differences in the raw Nyquist plots and the total grain boundary conductivities for each of these samples, Figs. 15 and 16, the specific grain boundary conductivities are, within experimental error, identical. The samples sintered at $1500{ }^{\circ} \mathrm{C}$ appear to have somewhat higher grain boundary conductivities than the others; however, this is an experimental artifact related to the high electrode impedance, discussed above. The activation energy for grain boundary conductivity and the $\log$ of the pre-exponential term for samples sintered in air (as averaged from samples sintered at 1400 and $1600{ }^{\circ} \mathrm{C}$ ) are $0.68(1) \mathrm{eV}$ and 3.8(4) $\Omega^{-1} \mathrm{~cm}^{-1} \mathrm{~K}$, respectively. The analogous values for samples sintered in oxygen are $0.73(1) \mathrm{eV}$ and 4.68(4) $\Omega^{-1} \mathrm{~cm}^{-1} \mathrm{~K}$.

Comparisons of the conductivity of polycrystalline barium cerate (as obtained from a batch-1 sample) in dry, $\mathrm{H}_{2} \mathrm{O}$-, and $\mathrm{D}_{2} \mathrm{O}$-saturated $\mathrm{Ar}$ in the bulk and grain boundary regions are presented in Fig. 21. The corresponding activation energies and pre-exponential terms are given in Table IV. The conversion from total grain boundary conductivity, $\sigma_{2}$, to specific grain boundary conductivity was carried out using the values of $G / g$ also given in Table IV. That this microstructural parameter was independent of atmosphere further supports the assumption that $\epsilon_{1} / \epsilon_{2}$ is primarily a measure of microstructural features, and, more generally, supports the validity of the brick layer model.

Impedance plots from barium cerate fibers obtained from the LHPG method exhibited only one arc, Fig. 22, indicating the absence of grain boundaries in a direction normal to the applied field, and presumably also parallel to it. Measurements were made such that the applied field was parallel to the growth direction. The Arrhenius plots obtained for the fiber conductivity in dry, $\mathrm{H}_{2} \mathrm{O}$-, and $\mathrm{D}_{2} \mathrm{O}$-saturated Ar are shown in Fig. 23; the corresponding activation energies and pre-exponential terms are given in Table V. Two fiber samples were examined, and it was noted that there was a significant difference in their electrical behavior, with the conductivity in $\mathrm{H}_{2} \mathrm{O}$ saturated argon of the first sample being about twice that of the second. These differences were primarily reflected in differences in the pre-exponential term, $\mathrm{A}$, while the activation energies in any atmosphere did not differ by more than $0.015 \mathrm{eV}$. The values provided in Table $\mathrm{V}$ represent the averages, whereas Fig. 24 shows the results for the more conductive sample.

Two key points are noteworthy from the results of Figs. 21 and 23. Firstly, there is a distinct isotope effect for all measurements, and secondly, the conductivity of the fibers is notably lower than that of the grain interiors. The isotope effect, manifested by a higher conductivity in $\mathrm{H}_{2} \mathrm{O}$ than $\mathrm{D}_{2} \mathrm{O}$-saturated atmospheres, arises from both classical and quantum mechanical consideration, as discussed by Nowick. ${ }^{6}$ Classical theory predicts, under the assumption that the potential well in which the mobile species resides is unchanged upon exchanging protons with deuterons, that the ratio of the proton and deuteron attempt frequencies is

$$
\frac{\omega_{H}}{\omega_{D}}=\frac{\sqrt{\frac{k}{m_{H}}}}{\sqrt{\frac{k}{m_{D}}}}=\sqrt{2}=1.41
$$

where $k$ is the force constant describing the potential well. Because the pre-exponential term, $A$, is directly proportional to the attempt frequency, $\omega$ (and this is the only manner in which $A$ is affected by the isotope exchange), then $A_{H} / A_{D}$ should also equal 1.41. Quantum mechanical theory predicts, again under the assumption that the potential well is unaffected by the isotope exchange, that the zero point energy of the mobile species is

$$
E_{0}=(3 / 2) h \omega,
$$


where $h$ is Planck's constant. The difference in zero point energies for protons and deuterons is then

$$
E_{0}^{H}-E_{0}^{D}=\left(\frac{3}{2}\right) h\left(\omega_{H}-\omega_{D}\right) \approx 0.44 h \omega_{H} .
$$

Assuming a typical vibrational frequency of 1 to $3 \times$ $10^{13} \mathrm{~s}^{-1}$, one obtains $\Delta E_{0}$ of 0.02 to $0.06 \mathrm{eV}$, and this, in turn, leads to a similar difference in activation energies for ion motion.

The observation of an isotope effect that is comparable in magnitude to that expected for the simple quantum mechanical treatment, Table VI, confirms that protons are the mobile species in the bulk (grain interiors), as is widely accepted, and, more significantly, demonstrates that protons are the mobile species in grain boundaries of barium cerate as well. It is noteworthy that the isotope effect on the pre-exponential term, $A$, is almost negligible, with $A_{H} / A_{D} \sim 1$ in all cases. Nowick, ${ }^{6}$ who noted similar nonclassical behavior in a number of compounds including $\mathrm{Nd}$-doped barium cerate, $\mathrm{Yb}$ doped strontium cerate, Sc-doped strontium zirconate, and nonstoichiometric strontium calcium niobate, has argued that $A_{D}>A_{H}$ will result if the migrating species, having acquired the energy to overcome an energy barrier between sites, retains a significant enough amount of energy such that it can transverse the barrier again, but in the reverse direction.

The low conductivity (and low activation energy) of the LHPG-grown fibers of barium cerate is undoubtedly a result of the low barium content and presence of $\mathrm{CeO}_{2}$ precipitates in these samples. Compositional variations can also explain the differences in behavior observed for the two fiber samples. Indeed, a comparison of the conductivity obtained for the first fiber sample with that of polycrystalline $\mathrm{Ba}_{0.96} \mathrm{Ce}_{0.85} \mathrm{Gd}_{0.15} \mathrm{O}_{3-\delta}{ }^{4}$ in a $\mathrm{H}_{2} \mathrm{O}$ saturated atmosphere, Fig. 24, shows the materials to have comparable electrical properties. Moreover, the dielectric constant obtained for the fibers was significantly greater than that of the (stoichiometric) polycrystalline materials, and such behavior has also been observed for barium-deficient polycrystalline samples. ${ }^{30}$
Alternatively, one might be tempted to consider the possibility that the absence of grain boundaries causes the high resistivity of the single-crystal samples. This is equivalent to assuming that $\sigma_{\text {bulk }}$ in Eq. (6) is equal to $\sigma_{\text {crystal }}$, and that $\sigma_{1}$ has a significant contribution from $\sigma_{g b}$. At $200{ }^{\circ} \mathrm{C}$ in an $\mathrm{H}_{2} \mathrm{O}$-saturated atmosphere the values of $\sigma_{1}$ and $\sigma_{2}$ for batch-1 samples were 0.16 and $0.83\left(\mathrm{k}^{-1} \mathrm{~cm}^{-1}\right.$, respectively, whereas the single-crystal fiber conductivity was $0.08(\mathrm{k} \Omega)^{-1} \mathrm{~cm}^{-1}$. Solving for $g / G$ [from Eqs. (6) and (7)] gives 0.21, implying that grain thickness should be $\sim 20 \%$ of the grain diameter (!) in order to account for both a high grain boundary resistance in series, as measured in terms of $\sigma_{2}$, and a high grain boundary conductivity in parallel, as measured in terms of $\sigma_{1}-\sigma_{\text {crystal }}$. Thus, something other than microstructural considerations must account for the differences in single crystal and bulk polycrystalline properties.

\section{Discussion}

The possibility that an amorphous phase may exist at the grain boundaries of proton conducting perovskites has been investigated by other authors in a few related material systems. The results of those studies are summarized in Table VII. It is apparent that the formation of such a phase is dependent on the exact host material, the dopant, and the dopant concentration. The presence of impurities and compositional differences introduced by differences in processing conditions also undoubtedly influence the nature of the grain boundaries, and may be responsible, in particular, for the different results reported for Yb-doped strontium cerate. Nevertheless, the bulk conductivities of these materials are comparable, and thus the electrical properties appear to be independent of the presence or absence of an amorphous intergranular phase.

While we have established that grain boundary conduction does not dominate the total conductivity of barium cerate, it is clear that the boundaries have very different properties from those of the grain interiors. A high grain boundary activation energy for transport in

TABLE VII. Results of TEM investigations of the grain boundary regions of proton conducting perovskites.

\begin{tabular}{ccccc}
\hline \hline Host material & Dopant & Concentration & Intergranular phase? & Reference \\
\hline $\mathrm{BaCeO}_{3}$ & $\mathrm{Nd}$ & $5 \%$ & no & 6 \\
& $\mathrm{Nd}$ & $10 \%$ & no & 6 \\
& $\mathrm{Nd}$ & $12 \%$ & yes & 6 \\
& $\mathrm{Gd}$ & $10 \%$ & no & 6 \\
& $\mathrm{Gd}$ & $10 \%$ & no & Present work \\
$\mathrm{SrCeO}_{3}$ & $\mathrm{Gd}$ & $15 \%$ & no & Present work, 24 \\
$\mathrm{SrCeO}_{3}$ & $\mathrm{Yb}$ & $5 \%$ & yes & 31 \\
\hline \hline
\end{tabular}

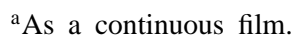

${ }^{\mathrm{b}}$ At triple point junctions. 
$\mathrm{H}_{2} \mathrm{O}$-saturated atmospheres has similarly been reported by Flint and Slade ${ }^{33}$ in 10\% Ca-doped barium cerate. For materials prepared by conventional solid state synthesis, an activation energy of 1.07(1) eV was obtained, and for those prepared from oxalate precursors, 0.73(4) $\mathrm{eV}$ was measured. In both cases, the bulk conductivity exhibited a typical activation energy of $\sim 0.64 \mathrm{eV}$. Stevenson et al. $^{34}$ have measured the bulk and boundary conductivities of $15 \%$ Gd-doped barium cerate. From their graphical data, the activation energy for grain boundary transport is $\sim 0.8 \mathrm{eV}$, where, again, the bulk conductivity has a typical value of $0.58 \mathrm{eV}$. Moreover, as both groups determined the grain boundary conductivity from Nyquist plots in which two arcs were observed, it is apparent that the specific grain boundary conductivity is lower than that of the bulk.

In order to explain the differences between the grain boundary and bulk properties of barium cerate, we propose (i) that a greater concentration of water molecules are dissolved into the grain boundary regions upon exposure to $\mathrm{H}_{2} \mathrm{O}$ than into the bulk, and (ii) that the oxygen ions and protons that form $\mathrm{OH}$ groups are more tightly bound together in the grain boundary regions than in the bulk. The first property explains the greater responsiveness of grain boundaries to a change in atmosphere from dry to wet argon and the second the high activation energy in the grain boundary region. As the grain boundary region contains, by definition, more structural defects than the bulk, it is quite reasonable to expect that in the dry state it should contain a large concentration of oxygen vacancies, and in the wet state a large concentration of hydroxyl groups. The grain interiors, in contrast, appear to contain hydroxyl groups even in the nominally dry state,${ }^{35}$ and dissolve a relatively small amount of $\mathrm{H}_{2} \mathrm{O}$ upon exposure to humid atmospheres. That the dissolution of water into the bulk must be low is supported by the observation that the unit cell dimensions of proton-conducting perovskites, within experimental error, are the same in the dry and wet states. ${ }^{31,36}$ Given the typical weight gain that polycrystalline samples undergo, however, it is impossible for the grain boundaries to account for the entirety of the $\mathrm{H}_{2} \mathrm{O}$ uptake. ${ }^{4} \mathrm{~A}$ high activation energy in the grain boundary region may be understood if one presumes, as has been recently proposed, ${ }^{37}$ that $\mathrm{O}-\mathrm{Ce}-\mathrm{O}$ bending vibrations are an essential step for the proton transport mechanism. Within the grain boundary region, disruption of the lattice will lead to disruption of such vibrational modes, and thus protons remain bound to specific oxygen ions.

Because the activation energy for grain boundary transport is higher than that for bulk transport, it may be expected that at some elevated temperature $\sigma_{g b}$ should be greater than $\sigma_{\text {bulk }}$, and accordingly, that transport along parallel grain boundaries may dominate $\sigma_{1}$. However, if we examine the ratio of $\sigma_{g b}$ to $\sigma_{\text {bulk }}$, for the barium cerate sintered in air, we obtain

$$
\frac{\sigma_{g b}}{\sigma_{\text {bulk }}}=0.08 \exp \left(\frac{-0.12 \mathrm{eV}}{k_{b} T}\right) \text {. }
$$

Thus, even at an infinitely high temperature the specific grain boundary conductivity is lower than the bulk. In terms of optimizing the transport properties of barium cerate, then, one merely needs to obtain grain large enough that the low frequency arc in the Nyquist plot becomes negligible with respect to the high frequency arc at the temperature of operation. For typical operating temperatures of $400-500{ }^{\circ} \mathrm{C}$, even a material with a grain size of $3 \mu \mathrm{m}$ meets the criterion. Enhancement of the transport properties of Gd-doped barium cerate by reducing the grain size is not possible, without a significant alternation of the grain boundary properties.

\section{Summary}

The results of this work can be summarized as follows:

(1) In the processing of doped barium cerate, a sintering temperature of approximately $1550{ }^{\circ} \mathrm{C}$ for a period of approximately $4 \mathrm{~h}$ yields a dense material. Higher sintering temperatures lead to grain boundary breakaway from pores, and thus larger grains but lower density. In addition, more severe sintering conditions (higher temperatures and/or longer times) leads to the loss of barium oxide. Highly barium-deficient materials will contain $\mathrm{CeO}_{2}$ precipitates and exhibit low conductivities and high dielectric constants.

(2) The brick layer model, which has been proven effective for the analysis of the properties of barium cerate, and several other materials by various authors, enables, one, if a two-arc spectrum is observed in the Nyquist plot of a polycrystalline material, and the material has a typical microstructure, to immediately conclude that the specific grain boundary resistance is greater than that of the bulk. Moreover, the specific grain boundary conductivity can be determined to relatively good precision from the electrical data alone, that is, in the absence of microstructural investigations.

(3) The grain boundaries in barium cerate are significantly more resistive that the bulk, and for similar processing routes, the specific grain boundary conductivity is independent of grain size. Moreover, the grain boundary region, even when free of an amorphous phase, is more responsive electrically to changes in atmosphere. These observations suggest that, after dissolution of $\mathrm{H}_{2} \mathrm{O}$ from the atmosphere, the concentration of hydroxyl groups is greater in the grain boundaries but that the protons in this region are more tightly bound to oxygen ions than they are in the bulk. The activation energy for proton transport in the grain boundary region of barium cerate is $0.69 \mathrm{eV}$ for samples sintered in air and $0.73 \mathrm{eV}$ 
for those sintered in oxygen. Under no conditions could one expect grain boundary transport along parallel grains to dominate the conduction process, unless, of course, the grain boundary properties are significantly altered by some other means.

\section{ACKNOWLEDGMENTS}

The authors thank Dr. Paul Carpenter (California Institute of Technology) and Dan Frech (University of Washington) for assistance with scanning electron microscopy and transmission electron microscopy, respectively. We also thank Geoff Staneff, who performed grain size measurements. Dr. Robert Feigelson (Stanford University) has kindly provided access to single crystal growth facilities. J.C. is grateful to Darryl Shima (formerly of the University of Washington) for guidance with experimental techniques. Funding of this work has been provided by the National Science Foundation through a National Young Investigator Award, and by the Electric Power Research Institute.

\section{REFERENCES}

1. H. Iwahara, T. Esaka, H. Uchida, and N. Maeda, Solid State Ionics 3/4, 359 (1981).

2. N. Bonanos, Solid State Ionics 53-56, 867 (1993).

3. H. Iwahara, Solid State Ionics 28-30, 573 (1988).

4. D. Shima and S. Haile, Solid State Ionics 97, 443 (1997).

5. K-D. Kreuer, E. Schönherr and J. Maier, in Proc. 14th Risø Intern. Symp. on Materials Science (Risø Natl. Lab., Roskilde, Denmark, 1993).

6. A. S. Nowick and Y. Du, Solid State Ionics 77, 137 (1995).

7. N. Bonanos, B. C. H. Steele and E. P. Butler, in Impedance Spectroscopy, edited by J.R. MacDonald (Wiley and Sons, New York, 1988), pp. 191-238.

8. H. Nafe, Solid State Ionics 13, 255 (1984).

9. X. Guo and R-Z. Yuan, J. Mater. Sci. Lett. 14, 499 (1995).

10. G. M. Christie and F. P.F. van Berkel, Solid State Ionics 83, 17 (1996).

11. J. R. MacDonald and W. B. Johnson, in Impedance Spectroscopy, edited by J.R. MacDonald (Wiley and Sons, New York, 1988), pp. $1-26$.
12. N. M. Beekmans and L. Heyne, Electrochim. Acta 21, 303 (1976).

13. T. van Dijk and A. J. Burggraaf, Phys. Status Solidi 63, 229 (1981).

14. M. Aoki, Y-M. Chiang, I. Kosacki, J-R. Lee, H.-L. Tuller, and Y. Liu, J. Am. Ceram. Soc. 75, 1169 (1996).

15. D. L. West, M.S. Thesis, University of Washington, Seattle (1996).

16. L. C. de Jonghe, J. Mater. Sci. 14, 33 (1979).

17. K. El. Adham and A. Hammou, Solid State Ionics 9 \& 10, 905 (1983).

18. M. J. Verkerk, B. J. Middlehuis, and A. J. Burggraaf, Solid State Ionics 6, 159 (1982).

19. J. C. Maxwell, A. Treatise on Electricity and Magnetism, 2nd ed. (Clarendon Press, Oxford, England, 1881).

20. J. E. Baurle, J. Phys. Chem. Solids 30, 2657 (1969).

21. E. Schouler, G. Giroud, and M. Kleitz, J. Chim. Phys. et Physicochim. Biol. 70, 1309 (1973).

22. K.W. Wagner, in Arkiv für Electrotechnik, edited by H. Schering (Springer-Verlag, Berlin, Germany, 1914).

23. D. West, S. M. Haile, and R. S. Feigelson, in Materials for Electrochemical Energy Storage and Conversion-Batteries, Capacitors and Fuel Cells, edited by D. H. Doughty, Brijesh Vyas, Tsutomu Takamura, and James R. Huff (Mater. Res. Soc. Symp. Proc. 393, Pittsburgh, PA, 1995) p. 31.

24. R. S. Feigelson, D. Gazit, D. K. Fork, and T.H. Geballe, Science 242, 1642 (1988).

25. J. T. Armstrong, Microbeam Analysis 4, 177 (1995).

26. B. B. Boukamp, Equivalent Circuit, University of Twente, The Netherlands (1988).

27. Micro-Cellref, from Materials Data, Inc., Livermore, CA (1992).

28. K. S. Knight and N. Bonanos, J. Mat. Chem. 4, 899 (1994).

29. W. D. Kingery, H. K. Bowen, and D. R. Uhlman, Introduction to Ceramics, 2nd ed. (John Wiley \& Sons, New York, 1976), pp. 469-490.

30. D. Shima and S. M. Haile, unpublished results.

31. F. Krug, T. Schober, R. Paul, and T. Springer, Solid State Ionics 77, 185 (1995).

32. J. Luyten, F. De Schutter, J. Schram, and J. Schoonman, Solid State Ionics 46, 117 (1991).

33. S. D. Flint and R. C. T. Glade, Solid State Ionics 77, 215 (1995).

34. D. A. Stevenson, N. Jiang, R. M. Buchanan, and F. E. G. Henn, Solid State Ionics 62, 279 (1993).

35. T. Scherban, Yu. M. Baikov, and E. K. Shalkova, Solid State Ionics 66, 159 (1993).

36. D. West and S. M. Haile, unpublished results.

37. K-D. Kreuer, Chem. Mater. 8, 610 (1996). 